\title{
ROTA-BAXTER ALGEBRAS, SINGULAR HYPERSURFACES, AND RENORMALIZATION ON KAUSZ COMPACTIFICATIONS
}

\author{
MATILDE MARCOLLI AND XIANG NI
}

\begin{abstract}
We consider Rota-Baxter algebras of meromorphic forms with poles along a (singular) hypersurface in a smooth projective variety and the associated Birkhoff factorization for algebra homomorphisms from a commutative Hopf algebra. In the case of a normal crossings divisor, the Rota-Baxter structure simplifies considerably and the factorization becomes a simple pole subtraction. We apply this formalism to the unrenormalized momentum space Feynman amplitudes, viewed as (divergent) integrals in the complement of the determinant hypersurface. We lift the integral to the Kausz compactification of the general linear group, whose boundary divisor is normal crossings. We show that the Kausz compactification is a Tate motive and that the boundary divisor and the divisor that contains the boundary of the chain of integration are mixed Tate configurations. The regularization of the integrals that we obtain differs from the usual renormalization of physical Feynman amplitudes, and in particular it may give mixed Tate periods in some cases that have non-mixed Tate contributions when computed with other renormalization methods.
\end{abstract}

\section{INTRODUCTION}

In this paper, we consider the problem of extracting periods of algebraic varieties from a class of divergent integrals arising in quantum field theory. The method we present here provides a regularization and extraction of finite values that differs from the usual (renormalized) physical Feynman amplitudes, but whose mathematical interest lies in the fact that it gives a period of a mixed Tate motive, for all graphs for which the amplitude can be computed using (global) forms with logarithmic poles. For more general graphs, one also obtains a period, where the nature of the motive involved depends on how a certain hyperplane arrangement intersects the big cell in a compactification of the general linear group. More precisely, the motive considered here is provided by the Kausz compactification of the general linear group and by a hyperplane arrangement that contains the boundary of the chain of integration. The regularization procedure we propose is modeled on the algebraic renormalization method, based on Hopf algebras of graphs and RotaBaxter algebras, as originally developed by Connes and Kreimer [22] and by Ebrahmi-Fard, Guo, and Kreimer [31. The main difference in our approach is that we apply the formalism to a RotaBaxter algebra of (even) meromorphic differential forms instead of applying it to a regularization of the integral. The procedure becomes especially simple in cases where the de Rham cohomology of the singular hypersurface complement is all realized by forms with logarithmic poles, in which case we replace the divergent integral with a family of convergent integrals obtained by a pole subtraction on the form and by (iterated) Poincaré residues. A similar approach was developed for integrals in configuration spaces by Ceyhan and the first author [21].

In Section 2 we introduce Rota-Baxter algebras of even meromorphic forms, along the lines of [21], and we formulate a general setting for extraction of finite values (regularization and renormalization) of divergent integrals modeled on algebraic renormalization applied to these Rota-Baxter algebras of differential forms.

In Section 3 we discuss the Rota-Baxter algebras of even meromorphic forms in the case of a smooth hypersurface $Y \subset X$. We show that, when restricted to forms with logarithmic poles, the Rota-Baxter operator becomes simply a derivation, and the Birkhoff factorization collapses to a simple pole subtraction, as in the case of log divergent graphs. We show that this simple pole 
subtraction can lead to too much loss of information about the unrenormalized integrand and we propose considering the additional information of the Poincaré residue and an additional integral associated to the residue.

In Section 4 we consider the case of singular hypersurfaces $Y \subset X$ given by a simple normal crossings divisor. We show that, in this case, the Rota-Baxter operator satisfies a simplified form of the RotaBaxter identity, which however is not just a derivation. We show that this modified identity still suffices to have a simple pole subtraction $\phi_{+}(\Gamma)=(1-T) \phi(\Gamma)$ in the Birkhoff factorization, even though the negative piece $\phi_{-}(\Gamma)$ becomes more complicated. Again, to avoid too much loss of information in passing from $\phi(\Gamma)$ to $\phi_{+}(\Gamma)$, we consider, in addition to the renormalized integral $\int_{\sigma} \phi_{+}(\Gamma)$, the collection of integrals of the form $\int_{\sigma \cap Y_{I}} \operatorname{Res}_{Y_{I}}(\phi(\Gamma))$, where $\operatorname{Res}_{Y_{I}}$ is the iterated Poincaré residue, [25], along the intersection $Y_{I}=\cap_{j \in I} Y_{j}$ of components of $Y$. These integrals are all periods of mixed Tate motives if $\left\{Y_{I}\right\}$ is a mixed Tate configuration, in the sense of [33]. We discuss the question of further generalizations to more general types of singularities, beyond the normal crossings case, via Saito's theory of forms with logarithmic poles [58, by showing that one can also define a Rota-Baxter structure on the Saito complex of forms with logarithmic poles.

In Section 5 we present our main application, which is a regularization (different from the physical one) of the Feynman amplitudes in momentum space, computed on the complement of the determinant hypersurface as in [4. Since the determinant hypersurface has worse singularities than what we need, we pull back the integral computation to the Kausz compactification [47] of the general linear group, where the boundary divisor that replaces the determinant hypersurface is a simple normal crossings divisor. We show that the motive of the Kausz compactification is Tate, and that the components of the boundary divisor form a mixed Tate configuration. We discuss how one can replace the form $\eta_{\Gamma}$ of the Feynman amplitude with a form with logarithmic poles. In general, this form is defined on the big cell of the Kausz compactification. For certain graphs, it is possible to show, using the mixed Hodge structure, that the form with logarithmic poles extends globally to the Kausz compactification, with poles along the boundary divisor.

\section{Rota-BAXter AlgEBras OF MEROMORPHIC FORMS}

We generalize the algebraic renormalization formalism to a setting based on Rota-Baxter algebras of algebraic differential forms on a smooth projective variety with poles along a hypersurface.

2.1. Rota-Baxter algebras. A Rota-Baxter algebra of weight $\lambda$ is a unital commutative algebra $\mathcal{R}$ over a field $K$ such that $\lambda \in K$, together with a linear operator $T: \mathcal{R} \rightarrow \mathcal{R}$ satisfying the Rota-Baxter identity

$$
T(x) T(y)=T(x T(y))+T(T(x) y)+\lambda T(x y) .
$$

For example, Laurent polynomials $\mathcal{R}=\mathbb{C}\left[z, z^{-1}\right]$ with $T$ the projection onto the polar part are a Rota-Baxter algebra of weight -1 .

The Rota-Baxter operator $T$ of a Rota-Baxter algebra of weight -1 , satisfying

$$
T(x) T(y)+T(x y)=T(x T(y))+T(T(x) y),
$$

determines a splitting of $\mathcal{R}$ into $\mathcal{R}_{+}=(1-T) \mathcal{R}$ and $T \mathcal{R}$, where $(1-T) \mathcal{R}$ and $T \mathcal{R}$ are not just vector spaces but algebras, because of the Rota-Baxter relation (2.2). The algebra $T \mathcal{R}$ is non-unital. In order to work with unital algebras, one defines $\mathcal{R}_{-}$to be the unitization of $T \mathcal{R}$, that is, $T \mathcal{R} \oplus K$ with multiplication $(x, t)(y, s)=(x y+t y+s x, t s)$. For an introduction to Rota-Baxter algebras we refer the reader to [38]. 
2.2. Rota-Baxter algebras of even meromorphic forms. Let $Y$ be a hypersurface in a projective variety $X$, with defining equation $Y=\{f=0\}$. We denote by $\mathcal{M}_{X}^{\star}$ the sheaf of meromorphic differential forms on $X$, and by $\mathcal{M}_{X, Y}^{\star}$ the subsheaf of meromorphic forms on with poles (of arbitrary order) along $Y$, that is, $\mathcal{M}_{X, Y}^{\star}=j_{*} \Omega_{U}^{1}$, where $U=X \backslash Y$ and $j: U \hookrightarrow X$ is the inclusion. Passing to global sections of $\mathcal{M}_{X, Y}^{\star}$ gives a graded-commutative algebra over the field of definition of the varieties $X$ and $Y$, which, for simplicity, we will still denote by $\mathcal{M}_{X, Y}^{\star}$. We can write forms $\omega \in \mathcal{M}_{X, Y}^{\star}$ as sums $\omega=\sum_{p \geq 0} \alpha_{p} / f^{p}$, where the $\alpha_{p}$ are holomorphic forms.

In particular, we consider forms of even degrees, so that $\mathcal{M}_{X, Y}^{\text {even }}$ is a commutative algebra under the wedge product.

Lemma 2.1. The commutative algebra $\mathcal{M}_{X, Y}^{\text {even }}$, together with the linear operator $T: \mathcal{M}_{X, Y}^{\text {even }} \rightarrow$ $\mathcal{M}_{X, Y}^{\text {even }}$ defined as the polar part

$$
T(\omega)=\sum_{p \geq 1} \alpha_{p} / f^{p}
$$

is a Rota-Baxter algebra of weight -1 .

Proof. For $\omega_{1}=\sum_{p \geq 0} \alpha_{p} / f^{p}$ and $\omega_{2}=\sum_{q \geq 0} \beta_{q} / f^{q}$, we have

$$
\begin{gathered}
T\left(\omega_{1} \wedge \omega_{2}\right)=\sum_{p \geq 0, q \geq 1} \frac{\alpha_{p} \wedge \beta_{q}}{f^{p+q}}+\sum_{p \geq 1, q \geq 0} \frac{\alpha_{p} \wedge \beta_{q}}{f^{p+q}}-\sum_{p \geq 1, q \geq 1} \frac{\alpha_{p} \wedge \beta_{q}}{f^{p+q}}, \\
T\left(T\left(\omega_{1}\right) \wedge \omega_{2}\right)=\sum_{p \geq 1, q \geq 0} \frac{\alpha_{p} \wedge \beta_{q}}{f^{p+q}} \\
T\left(\omega_{1} \wedge T\left(\omega_{2}\right)\right)=\sum_{p \geq 0, q \geq 1} \frac{\alpha_{p} \wedge \beta_{q}}{f^{p+q}}, \\
T\left(\omega_{1}\right) \wedge T\left(\omega_{2}\right)=\sum_{p \geq 1, q \geq 1} \frac{\alpha_{p} \wedge \beta_{q}}{f^{p+q}},
\end{gathered}
$$

so that (2.2) is satisfied.

Note that the restriction to even form is introduced only in order to ensure that the resulting RotaBaxter algebra is commutative, while (2.3) satisfies (2.2) regardless of the restriction on degrees.

Remark 2.2. Equivalently, we have the following description of the Rota-Baxter operator, which we will use in the following. The linear operator

$$
T(\omega)=\alpha \wedge \xi, \quad \text { for } \omega=\alpha \wedge \xi+\eta
$$

acting on forms $\omega=\alpha \wedge \xi+\eta$, with $\alpha$ a meromorphic form on $X$ with poles on $Y$ and $\xi$ and $\eta$ holomorphic forms on $X$, is a Rota-Baxter operator of weight -1 .

The Rota-Baxter identity is equivalently seen then as follows. For $\omega_{i}=\alpha_{i} \wedge \xi_{i}+\eta_{i}$, with $i=1,2$, we have

$$
T\left(\omega_{1} \wedge \omega_{2}\right)=(-1)^{\left|\alpha_{2}\right|\left|\xi_{1}\right|} \alpha_{1} \wedge \alpha_{2} \wedge \xi_{1} \wedge \xi_{2}+\alpha_{1} \wedge \xi_{1} \wedge \eta_{2}+(-1)^{\left|\eta_{1}\right|\left|\alpha_{2}\right|} \alpha_{2} \wedge \eta_{1} \wedge \xi_{2}
$$

while

$$
\begin{gathered}
T\left(T\left(\omega_{1}\right) \wedge \omega_{2}\right)=(-1)^{\left|\alpha_{2}\right|\left|\xi_{1}\right|} \alpha_{1} \wedge \alpha_{2} \wedge \xi_{1} \wedge \xi_{2}+\alpha_{1} \wedge \xi_{1} \wedge \eta_{2} \\
T\left(\omega_{1} \wedge T\left(\omega_{2}\right)\right)=(-1)^{\left|\alpha_{2}\right|\left|\xi_{1}\right|} \alpha_{1} \wedge \alpha_{2} \wedge \xi_{1} \wedge \xi_{2}+(-1)^{\left|\eta_{1}\right|\left|\alpha_{2}\right|} \alpha_{2} \wedge \eta_{1} \wedge \xi_{2}
\end{gathered}
$$

and

$$
T\left(\omega_{1}\right) \wedge T\left(\omega_{2}\right)=(-1)^{\left|\alpha_{2}\right|\left|\xi_{1}\right|} \alpha_{1} \wedge \alpha_{2} \wedge \xi_{1} \wedge \xi_{2},
$$

where all signs are positive if the forms are of even degree. Thus, the operator $T$ satisfies (2.2).

The proof automatically extends to the following slightly more general setting. 
Lemma 2.3. Let $\left(X_{\ell}, Y_{\ell}\right)$ for $\ell \geq 1$ be a collection of smooth projective varieties $X_{\ell}$ with hypersurfaces $Y_{\ell}$, all defined over the same field. Then the commutative algebra $\bigwedge_{\ell} \mathcal{M}_{X_{\ell}, Y_{\ell}}^{\text {even }}$ is a Rota-Baxter algebra of weight -1 with the polar projection operator $T$ determined by the $T_{\ell}$ on each $\mathcal{M}_{X_{\ell}, Y_{\ell}}^{\text {even }}$.

A similar setting was considered in Theorem 6.4 of [21].

2.3. Renormalization via Rota-Baxter algebras. In [22], the BPHZ renormalization procedure of perturbative quantum field theory was reinterpreted as a Birkhoff factorization of loops in the pro-unipotent group of characters of a commutative Hopf algebra of Feynman graphs. This procedure of algebraic renormalization was reformulated in more general and abstract terms in [31], using Hopf algebras and Rota-Baxter algebras.

We summarize here quickly the basic setup of algebraic renormalization. We refer the reader to [22], 23], [31], [52] for more details.

The Connes-Kreimer Hopf algebra of Feynman graphs $\mathcal{H}$ is a commutative, non-cocommutative, graded, connected Hopf algebra over $\mathbb{Q}$ associated to a given Quantum Field Theory (QFT). A theory is specified by assigning a Lagrangian and the corresponding action functional, which in turn determines which graphs occur as Feynman graphs of the theory. For instance, the only allowed valences of vertices in a Feynman graph are the powers of the monomials in the fields that appear in the Lagrangian. The generators of the Connes-Kreimer Hopf algebra of a given QFT are the 1PI Feynman graphs $\Gamma$ of the theory, namely those Feynman graphs that are 2-egde connected. As a commutative algebra, $\mathcal{H}$ is then just a polynomial algebra in the 1PI graphs $\Gamma$. A grading on $\mathcal{H}$ is given by the loop number (first Betti number) of graphs. In the case where Feynman graphs also have vertices of valence 2 , one uses the number of internal edges instead of loop number, to have finite dimensional graded pieces, but we ignore this subtlety for the present purposes. The grading satisfies

$$
\operatorname{deg}\left(\Gamma_{1} \cdots \Gamma_{n}\right)=\sum_{i} \operatorname{deg}\left(\Gamma_{i}\right), \quad \operatorname{deg}(1)=0 .
$$

The connectedness property means that the degree zero part is just $\mathbb{Q}$. The coproduct in $\mathcal{H}$ is given by

$$
\Delta(\Gamma)=\Gamma \otimes 1+1 \otimes \Gamma+\sum_{\gamma \in \mathcal{V}(\Gamma)} \gamma \otimes \Gamma / \gamma
$$

where the class $\mathcal{V}(\Gamma)$ consists of all (not necessarily connected) divergent subgraphs $\gamma$ such that the quotient graph (identifying each component of $\gamma$ to a vertex) is still a 1PI Feynman graph of the theory. As in any graded connected Hopf algebra, the antipode is constructed inductively as

$$
S(\Gamma)=-\Gamma-\sum S\left(\Gamma^{\prime}\right) \Gamma^{\prime \prime}
$$

for $\Delta(\Gamma)=\Gamma \otimes 1+1 \otimes \Gamma+\sum \Gamma^{\prime} \otimes \Gamma^{\prime \prime}$, with the terms $\Gamma^{\prime}, \Gamma^{\prime \prime}$ of lower degrees.

Remark 2.4. The general element in the Hopf algebra $\mathcal{H}$ is not a graph $\Gamma$ but a polynomial function $P=\sum a_{i_{1}, \ldots, i_{k}} \Gamma_{i_{1}}^{n_{i_{1}}} \cdots \Gamma_{i_{k}}^{n_{i_{k}}}$ with $\mathbb{Q}$ coefficients in the generators given by the graphs. However, for simplicity of notation, in the following we will just write $\Gamma$ to denote an arbitrary element of $\mathcal{H}$.

An algebraic Feynman rule $\phi: \mathcal{H} \rightarrow \mathcal{R}$ is a homomorphism of commutative algebras from the Hopf algebra $\mathcal{H}$ of Feynman graphs to a Rota-Baxter algebra $\mathcal{R}$ of weight -1 ,

$$
\phi \in \operatorname{Hom}_{\mathrm{Alg}}(\mathcal{H}, \mathcal{R}) .
$$

The set $\operatorname{Hom}_{\mathrm{Alg}}(\mathcal{H}, \mathcal{R})$ has a group structure, where the multiplication $\star$ is dual to the coproduct in the Hopf algebra, $\phi_{1} \star \phi_{2}(\Gamma)=\left\langle\phi_{1} \otimes \phi_{2}, \Delta(\Gamma)\right\rangle$.

Algebra homomorphisms $\phi: \mathcal{H} \rightarrow \mathcal{R}$ between a Hopf algebra $\mathcal{H}$ and a Rota-Baxter algebra $\mathcal{R}$ are also often referred to as "characters" in the renormalization literature. 
The morphism $\phi$ by itself does not know about the coalgebra structure of $\mathcal{H}$ and the Rota-Baxter structure of $\mathcal{R}$. These enter in the factorization of $\phi$ into divergent and finite part.

A Birkhoff factorization of an algebraic Feynman rule consists of a pair of commutative algebra homomorphisms

$$
\phi_{ \pm} \in \operatorname{Hom}_{\mathrm{Alg}}\left(\mathcal{H}, \mathcal{R}_{ \pm}\right)
$$

where $\mathcal{R}_{ \pm}$is the splitting of $\mathcal{R}$ induced by the Rota-Baxter operator $T$, with $\mathcal{R}_{+}=(1-T) \mathcal{R}$ and $\mathcal{R}_{-}$the unitization of $T \mathcal{R}$, satisfying

$$
\phi=\left(\phi_{-} \circ S\right) \star \phi_{+},
$$

with the product $\star$ dual to the coproduct $\Delta$ as above. The Birkhoff factorization is unique if one also imposes the normalization condition $\epsilon_{-} \circ \phi_{-}=\epsilon$, where $\epsilon$ is the counit of $\mathcal{H}$ and $\epsilon_{-}$is the augmentation in the algebra $\mathcal{R}_{-}$.

As shown in Theorem 4 of [22] (see equations (32) and (33) therein), there is an inductive formula for the Birkhoff factorization of an algebraic Feynman rule, of the form

$$
\phi_{-}(\Gamma)=-T\left(\phi(\Gamma)+\sum \phi_{-}\left(\Gamma^{\prime}\right) \phi\left(\Gamma^{\prime \prime}\right)\right) \quad \text { and } \quad \phi_{+}(\Gamma)=(1-T)\left(\phi(\Gamma)+\sum \phi_{-}\left(\Gamma^{\prime}\right) \phi\left(\Gamma^{\prime \prime}\right)\right)
$$

where $\Delta(\Gamma)=1 \otimes \Gamma+\Gamma \otimes 1+\sum \Gamma^{\prime} \otimes \Gamma^{\prime \prime}$.

The Birkhoff factorization (2.6) of algebra homomorphisms $\phi \in \operatorname{Hom}_{\mathrm{Alg}}(\mathcal{H}, \mathcal{R})$ is often referred to as "algebraic Birkhoff factorization", to distinguish it from the (analytic) Birkhoff factorization formulated in terms of loops (or infinitesimal loops) with values in Lie groups. We refer the reader to $\S 6.4$ of Chapter 1 of [23] for a discussion of the relation between these two kinds of Birkhoff factorization.

In the original Connes-Kreimer formulation, this approach is applied to the unrenormalized Feynman amplitudes regularized by dimensional regularization, with the Rota-Baxter algebra consisting of germs of meromorphic functions at the origin, with the operator of projection onto the polar part of the Laurent series.

In the following, we consider the following variant on the Hopf algebra of Feynman graphs.

Definition 2.5. As an algebra, $\mathcal{H}_{\text {even }}$ is the commutative algebra generated by Feynman graphs of a given scalar quantum field theory that have an even number of internal edges, $\# E(\Gamma) \in 2 \mathbb{N}$. The coproduct (2.5) on $\mathcal{H}_{\text {even }}$ is similarly defined with the sum over divergent subgraphs $\gamma$ with even $\# E(\gamma)$, with 1 PI quotient.

Notice that in dimension $D \in 4 \mathbb{N}$ all the log divergent subgraphs $\gamma \subset \Gamma$ have an even number of edges, since $D b_{1}(\gamma)=2 \# E(\gamma)$ in this case. This is a class of graphs that are especially interesting in physical applications.

Question 2.6. Is there a graded-commutative version of Birkhoff factorization involving gradedcommutative Rota-Baxter and Hopf algebras?

Such an extension to the graded-commutative case would be necessary to include the more general case of differential forms of odd degree (associated to Feynman graphs with an odd number of internal edges).

One can approach the question above by using the general setting of [32]:

(1) Let $\mathcal{H}$ be any connected filtered cograded Hopf algebra and let $\mathcal{R}$ be a (not necessarily commutative) associative algebra equipped with a Rota-Baxter operator of weight $\lambda \neq 0$. The algebraic Birkhoff factorization of any $\phi \in \operatorname{Hom}(\mathcal{H}, \mathcal{R})$ was obtained by Ebrahimi-Fard, Guo and Kreimer in 32 .

(2) However, if the target algebra $\mathcal{R}$ is not commutative, the set of characters $\operatorname{Hom}(\mathcal{H}, \mathcal{R})$ is not a group since it is not closed under convolution product, i.e. if $f, g \in \operatorname{Hom}(\mathcal{H}, \mathcal{R})$, then $f \star g$ does not necessarily belong to $\operatorname{Hom}(\mathcal{H}, \mathcal{R})$. 
The usual proof (see Theorem 4 of [22] and Theorem 1.39 in Chapter 1 of [23]) of the fact that the two parts $\phi_{ \pm}$of the Birkhoff factorization are algebra homomorphisms uses explicitly both the commutativity of the target Rota-Baxter algebra $\mathcal{R}$ and the fact that $\operatorname{Hom}_{\mathrm{Alg}}(\mathcal{H}, \mathcal{R})$ is a group, and does not extend directly to the graded-commutative case. The argument given in Theorems 3.4 and 3.7 of 32 provides a more general form of Birkhoff factorization that applies to a gradedcommutative (and more generally non-commutative) Rota-Baxter algebra. The resulting form of the factorization is more complicated than in the commutative case, in general. However, if the Rota-Baxter operator of weight -1 also satisfies $T^{2}=T$ and $T(T(x) y)=T(x) y$ for all $x, y \in \mathcal{R}$, then the form of the Birkhoff factorization for not necessarily commutative Rota-Baxter algebras simplifies considerably, and the $\phi_{+}$part of the factorization consists of a simple pole subtraction, as we prove in Proposition 2.10 below.

2.4. Rota-Baxter algebras and Atkinson factorization. In the following we will discuss some interesting properties of algebraic Birkhoff decomposition when the Rota-Baxter operator satisfies the identity $T(T(x) y)=T(x) y$.

Let $e: \mathcal{H} \rightarrow \mathcal{R}$ be the unit of $\operatorname{Hom}(\mathcal{H}, \mathcal{R})$ (under the convolution product) defined by $e\left(1_{\mathcal{H}}\right)=1_{\mathcal{R}}$ and $e(\Gamma)=0$ on $\oplus_{n>0} \mathcal{H}_{n}$.

The main observation can be summarized as follows:

(1) If the Rota-Baxter operator $T$ on $\mathcal{R}$ also satisfies the identity $T(T(x) y)=T(x) y$, then on $\operatorname{ker}(e)=\oplus_{n>0} \mathcal{H}_{n}$, the negative part of the Birkhoff factorization $\phi_{-}$takes the following form:

$$
\phi_{-}=-T(\phi(\Gamma))-\sum T\left(\phi\left(\Gamma^{\prime}\right)\right) \phi\left(\Gamma^{\prime \prime}\right), \quad \text { for } \Delta(\Gamma)=1 \otimes \Gamma+\Gamma \otimes 1+\sum \Gamma^{\prime} \otimes \Gamma^{\prime \prime} .
$$

(2) If $T$ also satisfies $T(x T(y))=x T(y), \forall x, y \in \mathcal{R}$, then the positive part is given by $\phi_{+}(\Gamma)=$ $(1-T)(\phi(\Gamma)), \forall \Gamma \in \operatorname{ker}(e)=\oplus_{n>0} \mathcal{H}_{n}$.

This follows from the properties of the Atkinson Factorization in Rota-Baxter algebras, which we recall below.

Proposition 2.7. (Atkinson Factorization, [7, see also [39]) Let $(\mathcal{R}, T)$ be a Rota-Baxter algebra of weight $\lambda \neq 0$. Let $\tilde{T}=-\lambda \mathrm{id}-T$ and let $a \in \mathcal{R}$. Assume that $b_{l}$ and $b_{r}$ are solutions of the fixed point equations

$$
b_{l}=1+T\left(b_{l} a\right), \quad b_{r}=1+\tilde{T}\left(a b_{r}\right) .
$$

Then

$$
b_{l}(1+\lambda a) b_{r}=1
$$

Thus

$$
1+\lambda a=b_{l}^{-1} b_{r}^{-1}
$$

if $b_{l}$ and $b_{r}$ are invertible.

A Rota-Baxter algebra $(\mathcal{R}, T)$ is called complete if there are algebras $\mathcal{R}_{n} \subseteq \mathcal{R}, n \geq 0$, such that $\left(\mathcal{R}, \mathcal{R}_{n}\right)$ is a complete algebra and $T\left(\mathcal{R}_{n}\right) \subseteq \mathcal{R}_{n}$.

Proposition 2.8. (Existence and uniqueness of the Atkinson Factorization, [39]) Let $\left(\mathcal{R}, T, \mathcal{R}_{n}\right)$ be a complete Rota-Baxter algebra of weight $\lambda \neq 0$. Let $\tilde{T}=-\lambda \mathrm{id}-T$ and let $a \in \mathcal{R}_{1}$.

(1) Equations (2.7) have unique solutions $b_{l}$ and $b_{r}$. Further $b_{l}$ and $b_{r}$ are invertible. Hence the Atkinson Factorization (2.8) exists.

(2) If $\lambda \neq 0$ and $T^{2}=-\lambda T$ (in particular if $T^{2}=-\lambda T$ on $\mathcal{R}$ ), then there are unique $c_{l} \in$ $1+T(\mathcal{R})$ and $c_{r} \in 1+\tilde{T}(\mathcal{R})$ such that

$$
1+\lambda a=c_{l} c_{r} .
$$


Define

$$
(T a)^{[n+1]}:=T\left((T a)^{[n]} a\right) \text { and }(T a)^{\{n+1\}}=T\left(a(T a)^{\{n\}}\right)
$$

with the convention that $(T a)^{[1]}=T(a)=(T a)^{\{1\}}$ and $(T a)^{[0]}=1=(T a)^{\{0\}}$.

Proposition 2.9. Let $\left(\mathcal{R}, \mathcal{R}_{n}, T\right)$ be a complete filtered Rota-Baxter algebra of weight -1 such that $T^{2}=T$. Let $a \in \mathcal{R}_{1}$. If $T$ also satisfies the following identity

$$
T(T(x) y)=T(x) y, \quad \forall x, y \in \mathcal{R},
$$

then the equation

$$
b_{l}=1+T\left(b_{l} a\right)
$$

has a unique solution

$$
1+T(a)(1-a)^{-1}
$$

Proof. First, we have $(T a)^{[n+1]}=T(a) a^{n}$ for $n \geq 0$. In fact, the case when $n=0$ just follows from the definition. Suppose it is true up to $n$, then $(T a)^{[n+2]}=T\left((T a)^{[n+1]} a\right)=T\left(\left(T(a) a^{n}\right) a\right)=$ $T\left(T(a) a^{n+1}\right)=T(a) a^{n+1}$. Arguing as in [32], $b_{l}=\sum_{n=0}^{\infty}(T a)^{[n]}=1+T(a)+T(T(a) a)+\cdots+$ $(T a)^{[n]}+\cdots$ is the unique solution of (2.10). So

$$
\begin{aligned}
b_{l} & =1+T(a)+T(a) a+T(a) a^{2}+\cdots \\
& =1+T(a)\left(1+a+a^{2}+\cdots\right) \\
& =1+T(a)(1-a)^{-1} .
\end{aligned}
$$

A bialgebra $\mathcal{H}$ over a field $K$ is called a connected, filtered cograded bialgebra if there are subspaces $\mathcal{H}_{n}$ of $\mathcal{H}$ such that $(a) \mathcal{H}_{p} \mathcal{H}_{q} \subseteq \sum_{k \leq p+q} \mathcal{H}_{k} ;(b) \Delta\left(\mathcal{H}_{n}\right) \subseteq \oplus_{p+q=n} \mathcal{H}_{p} \otimes \mathcal{H}_{q} ;(c) \mathcal{H}_{0}=\operatorname{im}(u)=K$, where $u: K \rightarrow \mathcal{H}$ is the unit of $\mathcal{H}$.

Proposition 2.10. Let $\mathcal{H}$ be a connected filtered cograded bialgebra (hence a Hopf algebra) and let $(\mathcal{R}, T)$ be a (not necessarily commutative) Rota-Baxter algebra of weight $\lambda=-1$ with $T^{2}=T$. Suppose that $T$ also satisfies (2.9). Let $\phi: \mathcal{H} \rightarrow \mathcal{R}$ be a character, that is, an algebra homomorphism. Then there are unique maps $\phi_{-}: \mathcal{H} \rightarrow T(\mathcal{R})$ and $\phi_{+}: \mathcal{H} \rightarrow \tilde{T}(\mathcal{R})$, where $\tilde{T}=1-T$, such that

$$
\phi=\phi_{-}^{*(-1)} * \phi_{+},
$$

where $\phi^{*(-1)}=\phi \circ S$, with $S$ the antipode. $\phi_{-}$takes the following form on $\operatorname{ker}(e)=\oplus_{n>0} \mathcal{H}_{n}$ :

$$
\begin{aligned}
\phi_{-}(\Gamma) & =-T(\phi(\Gamma))-\sum_{n=1}^{\infty}(-1)^{n} \sum T\left(\phi\left(\Gamma^{(1)}\right)\right) \phi\left(\Gamma^{(2)}\right) \phi\left(\Gamma^{(3)}\right) \cdots \phi\left(\Gamma^{(n+1)}\right) \\
& =-T(\phi(\Gamma))-\sum_{n=1}^{\infty}(-1)^{n}\left((T \phi) \tilde{*} \phi^{\tilde{*}^{n}}\right)(\Gamma) .
\end{aligned}
$$

Here we use the notation $\tilde{\Delta}^{n-1}(\Gamma)=\sum \Gamma^{(1)} \otimes \cdots \otimes \Gamma^{(n)}$, and $\tilde{\Delta}(\Gamma):=\Delta(\Gamma)-\Gamma \otimes 1-1 \otimes \Gamma$ (which is coassociative), and $\tilde{*}$ is the convolution product defined by $\tilde{\Delta}$. Furthermore, if $T$ satisfies

$$
T(x T(y))=x T(y), \quad \forall x, y \in A,
$$

then $\phi_{+}$takes the form on $\operatorname{ker}(e)=\oplus_{n>0} \mathcal{H}_{n}$ :

$$
\phi_{+}(\Gamma)=(1-T)(\phi(\Gamma)) .
$$


Proof. Define $R:=\operatorname{Hom}(\mathcal{H}, \mathcal{R})$ and

$$
P: R \rightarrow R, \quad P(f)(\Gamma)=T(f(\Gamma)), f \in \operatorname{Hom}(\mathcal{H}, \mathcal{R}), \Gamma \in \mathcal{H} .
$$

Then by [39], $R$ is a complete algebra with filtration $R_{n}=\left\{f \in \operatorname{Hom}(\mathcal{H}, \mathcal{R}) \mid f\left(\mathcal{H}_{n-1}\right)=0\right\}, n \geq 0$, and $P$ is a Rota-Baxter operator of weight -1 and $P^{2}=P$. Moreover, since $T$ satisfies (2.9), it is easy to check that $P(P(f) g)=P(f) g$ for any $f, g \in \operatorname{Hom}(\mathcal{H}, \mathcal{R})$. Let $\phi: \mathcal{H} \rightarrow \mathcal{R}$ be a character. Then $(e-\phi)\left(1_{\mathcal{H}}\right)=e\left(1_{\mathcal{H}}\right)-\phi\left(1_{\mathcal{H}}\right)=1_{\mathcal{R}}-1_{\mathcal{R}}=0$. So $e-\phi \in \mathcal{R}_{1}$. Set $a=e-\phi$, by Proposition 2.8, we know that there are unique $c_{l} \in T(\mathcal{R})$ and $c_{r} \in(1-T)(\mathcal{R})$ such that $\phi=c_{l} c_{r}$. Moreover, by Proposition 2.9 we have $\phi_{-}=b_{l}=c_{l}^{-1}=e+T(a)(e-a)^{-1}=e+T(e-\phi) \sum_{n=0}^{\infty}(e-\phi)^{n}$. We also have $\sum_{n=0}^{\infty}(e-\phi)^{n}\left(1_{\mathcal{H}}\right)=1_{\mathcal{R}}$ and for any $X \in \operatorname{ker}(e)=\oplus_{n>0} \mathcal{H}_{n}$, we have $(e-\phi)^{0}(\Gamma)=e(\Gamma)=0$; $(e-\phi)^{1}(\Gamma)=-\phi(\Gamma) ;(e-\phi)^{2}(\Gamma)=\sum(e-\phi)\left(\Gamma^{\prime}\right)(e-\phi)\left(\Gamma^{\prime \prime}\right)=\sum \phi\left(\Gamma^{\prime}\right) \phi\left(\Gamma^{\prime \prime}\right)$. More generally, we have $(e-\phi)^{n}(\Gamma)=(-1)^{n} \sum \phi\left(\Gamma^{(1)}\right) \phi\left(\Gamma^{(2)}\right) \cdots \phi\left(\Gamma^{(n)}\right)=(-1)^{n} \phi^{\tilde{*}^{n}}(\Gamma)$. So for $X \in \operatorname{ker}(e)=\oplus_{n>0} \mathcal{H}_{n}$,

$$
\begin{aligned}
\phi_{-}(\Gamma)= & \left(T(e-\phi) \sum_{n=0}^{\infty}(e-\phi)^{n}\right)(\Gamma) \\
= & T(e-\phi)\left(1_{\mathcal{H}}\right) \sum_{n=0}^{\infty}(e-\phi)^{n}(\Gamma)+T(e-\phi)(\Gamma) \sum_{n=0}^{\infty}(e-\phi)^{n}\left(1_{\mathcal{H}}\right) \\
& +\sum T\left((e-\phi)\left(\Gamma^{\prime}\right)\right) \sum_{n=1}^{\infty}(e-\phi)^{n}\left(\Gamma^{\prime \prime}\right) \\
= & -T(\phi(\Gamma))-\sum T\left(\phi\left(\Gamma^{\prime}\right)\right) \sum_{n=1}^{\infty}(-1)^{n} \sum \phi\left(\left(\Gamma^{\prime \prime}\right)^{(1)}\right) \phi\left(\left(\Gamma^{\prime \prime}\right)^{(2)}\right) \cdots \phi\left(\left(\Gamma^{\prime \prime}\right)^{(n)}\right) \\
= & -T(\phi(\Gamma))-\sum_{n=1}^{\infty}(-1)^{n} \sum T\left(\phi\left(\Gamma^{(1)}\right)\right) \phi\left(\Gamma^{(2)}\right) \phi\left(X^{(3)}\right) \cdots \phi\left(\Gamma^{(n+1)}\right) \\
= & -T(\phi(\Gamma))-\sum_{n=1}^{\infty}(-1)^{n}\left((T \phi)^{*} \phi^{*^{n}}\right)(\Gamma) .
\end{aligned}
$$

Suppose that $T$ also satisfies equation (2.11), then for any $a, b \in \mathcal{R}$, we have $(1-T)(a)(1-T)(b)=$ $a b-T(a) b-a T(b)+T(a) T(b)=a b-T(T(a) b)-T(a T(b))+T(a) T(b)=a b-T(a b)=(1-T)(a b)$, as $T$ is a Rota-Baxter operator of weight -1 . As shown in [22] and [32], $\phi_{+}(\Gamma)=(1-T)(\phi(\Gamma)+$ $\left.\sum \phi_{-}\left(\Gamma^{\prime}\right) \phi\left(\Gamma^{\prime \prime}\right)\right)$. So $\phi_{+}(\Gamma)=(1-T)(\phi(\Gamma))+\sum(1-T)\left(\phi_{-}\left(\Gamma^{\prime}\right)\right)(1-T)\left(\phi\left(\Gamma^{\prime \prime}\right)\right)$ by the previous computation. But $\phi_{-}$is in the image of $T$ and $T^{2}=T$, so we must have $(1-T)\left(\phi_{-}\left(\Gamma^{\prime}\right)\right)=0$, which shows that $\phi_{+}(\Gamma)=(1-T)(\phi(\Gamma))$.

2.5. A variant of algebraic renormalization. We consider now a setting inspired by the formalism of the Connes-Kreimer renormalization recalled above. The setting generalizes the one considered in [21] for configuration space integrals and our main application will be to extend the approach of [21] to momentum space integrals.

The main difference with respect to the Connes-Kreimer renormalization is that, instead of renormalizing the Feynman amplitude (regularized so that it gives a meromorphic function), we propose to renormalize the differential form, before integration, and then integrate the renormalized form to obtain a period.

The result obtained by this method differs from the physical renormalization, as we will discuss further in Section 5.11 below. There are at present no explicit examples of periods that are known not to be expressible in terms of rational combinations of mixed Tate periods, just because no such general statement of algebraic independence of numbers is known. However, it is generally expected that motives that are not mixed Tate will have periods that are not expressible in terms of mixed Tate periods, for instance periods associated to $H^{1}$ of an elliptic curve. There are known examples 
([18, [19]) of Feynman integrals that give periods of non-mixed Tate motives (a K3 surface, for instance). In our setting, the period obtained by applying the Birkhoff factorization to the Feynman integrand $\eta_{\Gamma}$ is always a mixed Tate period. However, it is difficult to ensure that the result is non-trivial. As we will discuss in more detail in Section 5, one can ensure a non-trivial result by replacing the form $\eta_{\Gamma}$ with a cohomologous form with logarithmic poles and taking into account both the result of the pole subtraction and all the Poincaré residues. However, passing to a form with logarithmic poles requires, in general, restricting to the big cell of the Kausz compactification, and this introduces a constraint on the nature of the period. If the intersection of the big cell of the Kausz compactification with the divisor $\Sigma_{\ell, g}$ that contains the boundary of the chain of integeration is a mixed Tate motive, then the convergent integral we obtain by replacing the integration form with a form with logarithmic poles is a mixed Tate period. For particular graphs, for which the form with logarithmic poles extends globally to the Kausz compactification, with poles along the boundary divisor, we obtain a mixed Tate period without any further assumption.

The main steps required for our setup are the following. For a variety $X$, we denote by $\mathfrak{m}(X)$ the motive in the Voevodsky category.

- For each $\ell \geq 1$, we construct a pair $\left(X_{\ell}, Y_{\ell}\right)$ of a smooth projective variety $X_{\ell}$ (defined over $\mathbb{Q})$ whose motive $\mathfrak{m}\left(X_{\ell}\right)$ is mixed Tate (over $\mathbb{Z}$ ), together with a (singular) hypersurface $Y_{\ell} \subset X_{\ell}$.

- For each Feynman graph $\Gamma$ with loop number $\ell$ we construct a map $\Upsilon: \mathbb{A}^{n} \backslash \hat{X}_{\Gamma} \rightarrow X_{\ell} \backslash Y_{\ell}$, where $\hat{X}_{\Gamma} \subset \mathbb{A}^{n}$ is the affine graph hypersurface, with $n$ the number of edges of $\Gamma$.

- Using the map $\Upsilon$, we describe the Feynman integrand as a morphism of commutative algebras

$$
\phi: \mathcal{H}_{\text {even }} \rightarrow \bigwedge_{\ell} \mathcal{M}_{X_{\ell}, Y_{\ell}}^{\text {even }}, \quad \phi(\Gamma)=\eta_{\Gamma},
$$

with $\mathcal{H}$ the Connes-Kreimer Hopf algebra and with the Rota-Baxter structure of Lemma 2.3 on the target algebra, and with $\eta_{\Gamma}$ an algebraic differential form on $X_{\ell}$ with polar locus $Y_{\ell}$, for $\ell=b_{1}(\Gamma)$.

- We express the (unrenormalized) Feynman integrals as a (generally divergent) integral $\int_{\Upsilon(\sigma)} \eta_{\Gamma}$, over a chain $\Upsilon(\sigma)$ in $X_{\ell}$ that is the image of a chain $\sigma$ in $\mathbb{A}^{n}$.

- We construct a divisor $\Sigma_{\ell} \subset X_{\ell}$, that contains the boundary $\partial \Upsilon(\sigma)$, whose motive $\mathfrak{m}\left(\Sigma_{\ell}\right)$ is mixed Tate (over $\mathbb{Z}$ ) for all $\ell \geq 1$.

- We perform the Birkhoff decomposition $\phi_{ \pm}$obtained inductively using the coproduct on $\mathcal{H}$ and the Rota-Baxter operator $T$ (polar part) on $\mathcal{M}_{X_{\ell}, Y_{\ell}}^{*}$.

- This gives a holomorphic form $\phi_{+}(\Gamma)$ on $X_{\ell}$. The divergent Feynman integral is then replaced by the integral

$$
\int_{\Upsilon(\sigma)} \phi_{+}(\Gamma)
$$

which is a period of the mixed Tate motive $\mathfrak{m}\left(X_{\ell}, \Sigma_{\ell}\right)$.

- In addition to the integral of $\phi_{+}(\Gamma)$ on $X_{\ell}$ we consider integrals on the strata of the complement $X_{\ell} \backslash Y_{\ell}$ of the polar part $\phi_{-}(\Gamma)$, which under suitable conditions will be interpreted as Poincaré residues.

If convergent, the Feynman integral $\int_{\Upsilon(\sigma)} \eta_{\Gamma}$ would be a period of $\mathfrak{m}\left(X_{\ell} \backslash Y_{\ell}, \Sigma_{\ell} \backslash\left(\Sigma_{\ell} \cap Y_{\ell}\right)\right)$. The renormalization procedure described above replaces it with a (convergent) integral that is a period of the simpler motive $\mathfrak{m}\left(X_{\ell}, \Sigma_{\ell}\right)$. By our assumptions on $X_{\ell}$ and $\Sigma_{\ell}$, the motive $\mathfrak{m}\left(X_{\ell}, \Sigma_{\ell}\right)$ is mixed Tate for all $\ell$.

Thus, this strategy eliminates the difficulty of analyzing the motive $\mathfrak{m}\left(X_{\ell} \backslash Y_{\ell}, \Sigma_{\ell} \backslash\left(\Sigma_{\ell} \cap Y_{\ell}\right)\right)$ encountered for instance in [4]. The form of renormalization proposed here always produces a mixed Tate period, but at the cost of incurring in a considerable loss of information with respect to the original Feynman integral. 
Indeed, a difficulty in the procedure described above is ensuring that the resulting regularized form

$$
\phi_{+}(\Gamma)=(1-T)\left(\phi(\Gamma)+\sum_{\gamma \subset \Gamma} \phi_{-}(\gamma) \wedge \phi(\Gamma / \gamma)\right)
$$

is nontrivial. This condition may be difficult to control in explicit cases, although we will discuss below (see Section 5) conditions under which one can reduce the problem to forms with logarithmic poles, where using the pole subtraction together with Poincaré residues one can obtain nontrivial periods (although the result one obtains is not equivalent to the physical renormalization of the Feynman amplitude).

An additional difficulty that can cause loss of information with respect to the Feynman integral is coming from the combinatorial conditions on the graph given in [4 that we will use to ensure that the map $\Upsilon$ to the complement of the determinant hypersurface is an embedding, see Section 5.11.

\section{Rota-Baxter Algebras AND FORMS With LOGARITHMiC POlES}

We now focus on the case of meromorphic forms with logarithmic poles, where the Rota-Baxter structure and the renormalization procedure described above drastically simplify.

Lemma 3.1. Let $X$ be a smooth projective variety and $Y \subset X$ a smooth hypersurface with defining equation $Y=\{f=0\}$. Let $\Omega_{X}^{\star}(\log (Y))$ be the sheaf of algebraic differential forms on $X$ with logarithmic poles along $Y$. After passing to global sections, we obtain a graded-commutative algebra, which we still denote by $\Omega_{X}^{\star}(\log (Y))$, for simplicity. The Rota-Baxter operator $T$ of Lemma 2.1] preserves the commutative subalgebra $\Omega_{X}^{\mathrm{even}}(\log (Y))$ and the pair $\left(\Omega_{X}^{\mathrm{even}}(\log (Y)), T\right)$ is a graded Rota-Baxter algebra of degree -1 with the property that, for all $\omega_{1}, \omega_{2} \in \Omega_{X}^{\text {even }}(\log (Y))$, the wedge product $T\left(\omega_{1}\right) \wedge T\left(\omega_{2}\right)=0$.

Proof. Forms $\omega \in \Omega_{X}^{\star}(\log (Y))$ can be written in canonical form

$$
\omega=\frac{d f}{f} \wedge \xi+\eta
$$

with $\xi$ and $\eta$ holomorphic, so that $T(\omega)=\frac{d f}{f} \wedge \xi$. We then have (2.2) as in Remark 2.2 above, with $T\left(\omega_{1}\right) \wedge T\left(\omega_{2}\right)=(-1)^{\left|\xi_{1}\right|+1} \alpha \wedge \alpha \wedge \xi_{1} \wedge \xi_{2}$ where $\alpha$ is the 1 -form $\alpha=d f / f$ so that $\alpha \wedge \alpha=0$.

Lemma 3.1 shows that, when restricted to $\Omega_{X}^{\star}(\log (Y))$, the operator $T$ satisfies the simpler identity

$$
T(x y)=T(T(x) y)+T(x T(y)) .
$$

This property greatly simplifies the decomposition of the algebra induced by the Rota-Baxter operator.

Let $\mathcal{R}_{+}=(1-T) \mathcal{R}$. For an operator $T$ satisfying (3.1) and $T(x) T(y)=0$, for all $x, y \in \mathcal{R}$, the property that $\mathcal{R}_{+} \subset \mathcal{R}$ is a subalgebra follows immediately from the simple identity

$$
\begin{gathered}
(1-T)(x) \cdot(1-T)(y)=x y-T(x) y-x T(y) \\
=x y-T(x) y-x T(y)-(T(x y)-T(T(x) y)-T(x T(y)))=(1-T)(x y-T(x) y-x T(y)) .
\end{gathered}
$$

Moreover, we obtain a simplified form of the general result of Proposition 2.10, when taking into account the vanishing $T(x) T(y)=0$, as shown in Lemma 3.1 .

Lemma 3.2. Let $\mathcal{R}$ be a commutative algebra and $T: \mathcal{R} \rightarrow \mathcal{R}$ a linear operator that satisfies the identity (3.1) and such that, for all $x, y \in \mathcal{R}$, the product $T(x) T(y)=0$. Then both $T$ and $1-T$ are idempotent, $T^{2}=T$ and $(1-T)^{2}=1-T$.

Proof. The identity (3.1) gives $T(1)=0$, since taking $x=y=1$ one obtains $T(1)=2 T^{2}(1)$ while taking $x=T(1)$ and $y=1$ gives $T^{2}(1)=T^{3}(1)$. Then (3.1) with $y=1$ gives $T(x)=T(x T(1))+$ $T(T(x) 1)=T^{2}(x)$ for all $x \in \mathcal{R}$. For $1-T$ we then have $(1-T)^{2}(x)=x-2 T(x)+T^{2}(x)=(1-T)(x)$, for all $x \in \mathcal{R}$. 
Lemma 3.3. Let $\mathcal{R}$ be a commutative algebra and $T: \mathcal{R} \rightarrow \mathcal{R}$ a linear operator that satisfies the identity (3.1) and such that, for all $x, y \in \mathcal{R}$ the product $T(x) T(y)=0$. If, for all $x, y \in \mathcal{R}$, the identity $T(x) y+x T(y)=T(T(x) y)+T(x T(y))$ holds, then the operator $(1-T): \mathcal{R} \rightarrow \mathcal{R}_{+}$is an algebra homomorphism and the operator $T$ is a derivation on $\mathcal{R}$.

Proof. We have $(1-T)(x y)=x y-T(T(x) y)-T(x T(y))$ while $(1-T)(x) \cdot(1-T)(y)=x y-$ $T(x) y-x T(y)$. Assuming that, for all $x, y \in \mathcal{R}$, we have $T(T(x) y)+T(x T(y))=T(x) y+x T(y)$ gives $(1-T)(x y)=(1-T)(x) \cdot(1-T)(y)$. Moreover, the identity (3.1) can be rewritten as $T(x y)=T(x) y+x T(y)$, hence $T$ is just a derivation on $\mathcal{R}$.

Consider then the case of a smooth hypersurface $Y$ in a smooth projective variety $X$. We have the following properties.

Proposition 3.4. Let $Y \subset X$ be a smooth hypersurface in a smooth projective variety. The RotaBaxter operator $T: \mathcal{M}_{X, Y}^{\text {even }} \rightarrow \mathcal{M}_{X, Y}^{\text {even }}$ of weight -1 on meromorphic forms on $X$ with poles along $Y$ restricts to a derivation on the graded algebra $\Omega_{X}^{\mathrm{even}}(\log (Y))$ of forms with logarithmic poles. Moreover, the operator $1-T$ is a morphism of commutative algebras from $\Omega_{X}^{\text {even }}(\log (Y))$ to the algebra of holomorphic forms $\Omega_{X}^{\mathrm{even}}$.

Proof. It suffices to check that the polar part operator $T: \Omega_{X}^{\text {even }}(\log (Y)) \rightarrow \Omega_{X}^{\text {even }}(\log (Y))$ satisfies the hypotheses of Lemma 3.3. We have seen that, for all $\omega_{1}, \omega_{2} \in \Omega_{X}^{\operatorname{even}}(\log (Y))$, the product $T\left(\omega_{1}\right) \wedge T\left(\omega_{2}\right)=0$. Moreover, for $\omega_{i}=d \log (f) \wedge \xi_{i}+\eta_{i}$, we have $T\left(\omega_{1}\right) \wedge \omega_{2}=d \log (f) \wedge \xi_{1} \wedge \eta_{2}$ and $\omega_{1} \wedge T\left(\omega_{2}\right)=(-1)^{\left|\eta_{1}\right|} d \log (f) \wedge \eta_{1} \wedge \xi_{2}$, where the $\xi_{i}$ and $\eta_{i}$ are holomorphic, so that we have $T\left(T\left(\omega_{1}\right) \wedge \omega_{2}\right)=T\left(\omega_{1}\right) \wedge \omega_{2}$ and $T\left(\omega_{1} \wedge T\left(\omega_{2}\right)\right)=\omega_{1} \wedge T\left(\omega_{2}\right)$. Thus, the hypotheses of Lemma 3.3 are satisfied.

3.1. Birkhoff factorization and forms with logarithmic poles. In cases where the pair $(X, Y)$ has the property that all de Rham cohomology classes in $H_{d R}^{*}(X \backslash Y)$ are represented by global algebraic differential forms with logarithmic poles, the construction above simplifies significantly. Indeed, the Birkhoff factorization becomes essentially trivial, because of Proposition 3.4. In other words, all graphs behave "as if they were log divergent". This can be stated more precisely as follows.

Proposition 3.5. Let $Y \subset X$ be a smooth hypersurface inside a smooth projective variety and let $\Omega_{X}^{\text {even }}(\log (Y))$ denote the commutative algebra of algebraic differential forms on $X$ of even degree with logarithmic poles on $Y$. Let $\phi: \mathcal{H} \rightarrow \Omega_{X}^{\mathrm{even}}(\log (Y))$ be a morphism of commutative algebras from a commutative Hopf algebra $\mathcal{H}$ to $\Omega_{X}^{\mathrm{even}}(\log (Y))$ with the operator $T$ of pole subtraction. Then for every $\Gamma \in \mathcal{H}$ one has

$$
\phi_{+}(\Gamma)=(1-T) \phi(\Gamma),
$$

while the negative part of the Birkhoff factorization takes the form

$$
\phi_{-}(\Gamma)=-T(\phi(\Gamma))-\sum \phi_{-}\left(\Gamma^{\prime}\right) \phi\left(\Gamma^{\prime \prime}\right),
$$

where $\Delta(\Gamma)=\Gamma \otimes 1+1 \otimes \Gamma+\sum \Gamma^{\prime} \otimes \Gamma^{\prime \prime}$. Moreover, $\phi_{-}$takes the following nonrecursive form on $\operatorname{ker}(e)=\oplus_{n>0} \mathcal{H}_{n}$ :

$$
\begin{aligned}
\phi_{-}(\Gamma) & =-T(\phi(\Gamma))-\sum_{n=1}^{\infty}(-1)^{n} \sum T\left(\phi\left(\Gamma^{(1)}\right)\right) \phi\left(\Gamma^{(2)}\right) \phi\left(\Gamma^{(3)}\right) \cdots \phi\left(\Gamma^{(n+1)}\right) \\
& =-T(\phi(\Gamma))-\sum_{n=1}^{\infty}(-1)^{n}\left((T \phi) \tilde{*} \phi^{\tilde{*}^{n}}\right)(\Gamma) .
\end{aligned}
$$


Proof. The operator $T$ of pole subtraction is a derivation on $\Omega_{X}^{\mathrm{even}}(\log (Y))$. By (2.6) we have $\phi_{+}(\Gamma)=(1-T)\left(\phi(\Gamma)+\sum \phi_{-}\left(\Gamma^{\prime}\right) \phi\left(\Gamma^{\prime \prime}\right)\right)$. By Proposition 3.4 we know that, in the case of forms with logarithmic poles along a smooth hypersurface, $1-T$ is an algebra homomorphism, hence $\left.\phi_{+}(\Gamma)=(1-T)(\phi(\Gamma))+\sum(1-T)\left(\phi_{-}\left(\Gamma^{\prime}\right)\right)(1-T)\left(\phi\left(\Gamma^{\prime \prime}\right)\right)\right)$, but $\phi_{-}\left(\Gamma^{\prime}\right)$ is in the range of $T$ and, again by Proposition 3.4, we have $T^{2}=T$, so that the terms in the sum all vanish, since $(1-T)\left(\phi_{-}\left(\Gamma^{\prime}\right)\right)=$ 0 . By (2.6) we have $\phi_{-}(\Gamma)=-T\left(\phi(\Gamma)+\sum \phi_{-}\left(\Gamma^{\prime}\right) \phi\left(\Gamma^{\prime \prime}\right)\right)=-T \phi(\Gamma)-\sum T\left(\phi_{-}\left(\Gamma^{\prime}\right)\right) \phi\left(\Gamma^{\prime \prime}\right)-$ $\sum \phi_{-}\left(\Gamma^{\prime}\right) T\left(\phi\left(X \Gamma^{\prime}\right)\right)$, because by Proposition $3.4 T$ is a derivation. The last sum vanishes because $\phi_{-}\left(\Gamma^{\prime}\right)$ is in the range of $T$ and we have $T(\eta) \wedge T(\xi)=0$ for all $\eta, \xi \in \Omega_{X}^{*}(\log (Y))$. Thus, we are left with $\phi_{-}(\Gamma)=-T \phi(\Gamma)-\sum T\left(\phi_{-}\left(\Gamma^{\prime}\right)\right) \phi\left(\Gamma^{\prime \prime}\right)=-T \phi(\Gamma)-\sum \phi_{-}\left(\Gamma^{\prime}\right) \phi\left(\Gamma^{\prime \prime}\right)$. The last part follows from Proposition 2.10, since $T(T(\eta) \wedge \xi)=T(\eta) \wedge \xi$.

Notice that this is compatible with the property that $\phi(\Gamma)=\left(\phi_{-} \circ S \star \phi_{+}\right)(\Gamma)$ (with the $\star$-product dual to the Hopf algebra coproduct). In fact, this identity is equivalent to $\phi_{+}=\phi_{-} \star \phi_{\text {, which }}$ means that $\phi_{+}(\Gamma)=\left\langle\phi_{-} \otimes \phi, \Delta(\Gamma)\right\rangle=\phi_{-}(\Gamma)+\phi(\Gamma)+\sum \phi_{-}\left(\Gamma^{\prime}\right) \phi\left(\Gamma^{\prime \prime}\right)=(1-T) \tilde{\phi}(\Gamma)$ as above. Equivalently, all the nontrivial terms $\phi_{-}\left(\Gamma^{\prime}\right) \phi\left(\Gamma^{\prime \prime}\right)$ in $\tilde{\phi}(\Gamma)$ satisfy $T\left(\phi_{-}\left(\Gamma^{\prime}\right) \phi\left(\Gamma^{\prime \prime}\right)\right)=\phi_{-}\left(\Gamma^{\prime}\right) \phi\left(\Gamma^{\prime \prime}\right)$, because of the simplified form (3.1) of the Rota-Baxter identity.

Corollary 3.6. Suppose given a character $\phi: \mathcal{H} \rightarrow \Omega_{X}^{\mathrm{even}}(\log (Y))$ of the Hopf algebra of Feynman graphs, where $X=X_{\ell}$ and $Y=Y_{\ell}$ independently of the number of loops $\ell \geq 1$. Then the negative part of the Birkhoff factorization of Proposition 3.5 has the simple form

$$
\phi_{-}(\Gamma)=-\frac{d h}{h} \wedge\left(\xi_{\Gamma}+\sum_{N \geq 1}(-1)^{N} \sum_{\gamma_{N} \subset \cdots \subset \gamma_{1} \subset \gamma_{0}=\Gamma} \xi_{\gamma_{N}} \wedge \bigwedge_{j=1}^{N} \eta_{\gamma_{j-1} / \gamma_{j}}\right),
$$

where $\phi(\Gamma)=\frac{d h}{h} \wedge \xi_{\Gamma}+\eta_{\Gamma}$, and $Y=\{h=0\}$.

Proof. The result follows from the expression

$$
\phi_{-}(\Gamma)=-T(\phi(\Gamma))-\sum_{\gamma \subset \Gamma} \phi_{-}(\gamma) \phi(\Gamma / \gamma)
$$

obtained in Proposition 3.5, where $\phi(\Gamma)=\omega_{\Gamma}=\frac{d h}{h} \wedge \xi_{\Gamma}+\eta_{\Gamma}$, so that $T(\phi(\Gamma))=\frac{d h}{h} \wedge \xi_{\Gamma}$ and $\phi(\Gamma / \gamma)=\frac{d h}{h} \wedge \xi_{\Gamma / \gamma}+\eta_{\Gamma / \gamma}$. The wedge product of $\phi_{-}(\gamma)=-T(\phi(\gamma))-\sum_{\gamma_{2} \subset \gamma} \phi_{-}\left(\gamma_{2}\right) \phi\left(\gamma / \gamma_{2}\right)$ with $\phi(\Gamma / \gamma)$ will give a term $\frac{d h}{h} \wedge \xi_{\gamma} \wedge \eta_{\Gamma / \gamma}$ and additional terms $\phi_{-}\left(\gamma_{2}\right) \phi\left(\gamma / \gamma_{2}\right) \wedge \eta_{\Gamma / \gamma}$. Proceeding inductively on these terms, one obtains (3.2).

Remark 3.7. In the geometric construction we consider here, one does not have a single pair $(X, Y)$ for all loop numbers. Instead, we consider a more general situation, where $X_{\ell}$ and $Y_{\ell}$ depend on the loop number $\ell \geq 1$. In this case, the form of the negative piece $\phi_{-}(\Gamma)$ is more complicated than in Corollary 3.6, as it contains forms on the products $X_{\ell(\gamma)} \times X_{\ell(\Gamma / \gamma)}$ with logarithmic poles along $Y_{\ell(\gamma)} \times X_{\ell(\Gamma / \gamma)} \cup X_{\ell(\gamma)} \times Y_{\ell(\Gamma / \gamma)}$. However, the general form of the expression is similar, only more cumbersome to write explicitly.

3.2. Polar subtraction and the residue. We have seen that, in the case of a smooth hypersurface $Y \subset X$, the Birkhoff factorization in the algebra of forms with logarithmic poles reduces to a simple pole subtraction, $\phi_{+}(\Gamma)=(1-T) \phi(\Gamma)$. If the unrenormalized $\phi(\Gamma)$ is a form written as $\alpha+\frac{d f}{f} \wedge \beta$, with $\alpha$ and $\beta$ holomorphic, then $\phi_{+}(\Gamma)$ vanishes identically whenever $\alpha=0$. In that case, all information about $\phi(\Gamma)$ is lost in the process of pole substraction. Suppose that $\int_{\sigma} \phi(\Gamma)$ is the 
original unrenormalized integral. To maintain some additional information, it is preferable to consider, in addition to the integral $\int_{\sigma} \phi_{+}(\Gamma)$, also an integral of the form

$$
\int_{\sigma \cap Y} \operatorname{Res}_{Y}(\eta)
$$

where $\operatorname{Res}_{Y}(\eta)=\beta$ is the Poincaré residue of $\eta=\alpha+\frac{d f}{f} \wedge \beta$ along $Y$. It is dual to the Leray coboundary, in the sense that

$$
\int_{\sigma \cap Y} \operatorname{Res}_{Y}(\eta)=\frac{1}{2 \pi i} \int_{\mathcal{L}(\sigma \cap Y)} \eta,
$$

where the Leray coboundary $\mathcal{L}(\sigma \cap Y)$ is a circle bundle over $\sigma \cap Y$. In this way, even when $\alpha=0$, one can still retain the nontrivial information coming from the Poincaré residue, which is also expressed as a period.

\section{Singular HYPERSURFACES AND MEROMORPHIC FORMS}

In our main application, we will need to work with pairs $(X, Y)$ where $X$ is smooth projective, but the hypersurface $Y$ is singular. Thus, we now discuss extensions of the results above to more general situations where $Y \subset X$ is a singular hypersurface in a smooth projective variety $X$.

Again we denote by $\mathcal{M}_{X, Y}^{*}$ the sheaf of meromorphic differential forms on $X$ with poles along $Y$, of arbitrary order, and by $\Omega_{X}^{*}(\log (Y))$ the sub-sheaf of forms with logarithmic poles along $Y$. Let $h$ be a local determination of $Y$, so that $Y=\{h=0\}$. We can then locally represent forms $\omega \in \mathcal{M}_{X, Y}^{*}$ as finite sums $\omega=\sum_{p>0} \omega_{p} / h^{p}$, with the $\omega_{p}$ holomorphic. The polar part operator $T: \mathcal{M}_{X, Y}^{\text {even }} \rightarrow \mathcal{M}_{X, Y}^{\text {even }}$ can then be defined as in (2.3).

In the case we considered in the previous section, with $Y \subset X$ a smooth hypersurface, forms with logarithmic poles can be represented as

$$
\omega=\frac{d h}{h} \wedge \xi+\eta
$$

with $\xi$ and $\eta$ holomorphic. The Leray residue is given by $\operatorname{Res}(\omega)=\xi$. It is well defined, as the restriction of $\xi$ to $Y$ is independent of the choice of a local equation for $Y$.

In the next subsection we discuss how the case of a smooth hypersurface generalizes to the case of a normal crossings divisor $Y \subset X$ inside a smooth projective variety $X$. The normal crossings divisor is a particularly nice case of a larger class of singular hypersurfaces. The complex of forms with logarithmic poles extends from the smooth hypersurface case to the normal crossings divisor case as in 25]. For more general singular hypersurfaces, an appropriate notion of forms with logarithmic poles was introduced by Saito in [58]. The construction of the residue was also generalized from the smooth hypersurface case to the case where $Y$ is a normal crossings divisor in [25] and to more general singular hypersurfaces in [58].

4.1. Normal crossings divisors. The main case of singular hypersurfaces that we focus on for our applications will be simple normal crossings divisors. In fact, while our formulation of the Feynman amplitude in momentum space is based on the formulation of [4, where the unrenormalized Feynman integral lives on the complement of the determinant hypersurface, which has worse singularities, we will reformulate the integral on the Kausz compactification of $\mathrm{GL}_{n}$ where the boundary divisor of the compactification is normal crossings.

If $Y \subset X$ is a simple normal crossings divisor in a smooth projective variety, with $Y_{j}$ the components of $Y$, with local equations $Y_{j}=\left\{f_{j}=0\right\}$, the complex of forms with logarithmic poles $\Omega_{X}^{*}(\log (Y))$ is spanned by the forms $\frac{d f_{j}}{f_{j}}$ and by the holomorphic forms on $X$. 
As in Theorem 6.3 of [21], we obtain that the Rota-Baxter operator of polar projection $T: \mathcal{M}_{X, Y}^{\text {even }} \rightarrow$ $\mathcal{M}_{X, Y}^{\text {even }}$ restricts to a Rota-Baxter operator $T: \Omega_{X}^{\text {even }}(\log (Y)) \rightarrow \Omega_{X}^{\text {even }}(\log (Y))$ given by

$$
T: \eta \mapsto T(\eta)=\sum_{j} \frac{d f_{j}}{f_{j}} \wedge \operatorname{Res}_{Y_{j}}(\eta),
$$

where the holomorphic form $\operatorname{Res}_{Y_{j}}(\eta)$ is the Poincaré residue of $\eta$ restricted to $Y_{j}$.

Unlike the case of a single smooth hypersurface, for a simple normal crossings divisor the RotaBaxter operator operator $T$ does not satisfy $T(x) T(y) \equiv 0$, since we now have terms like $\frac{d f_{j}}{f_{j}} \wedge \frac{d f_{k}}{f_{k}} \neq 0$, for $j \neq k$, so the Rota-Baxter identity for $T$ does not reduce to a derivation, but some of the properties that simplify the Birkhoff factorization in the case of a smooth hypersurface still hold in this case.

Proposition 4.1. The Rota-Baxter operator $T$ of (4.2) satisfies $T^{2}=T$ and the Rota-Baxter identity simplifies to the form

$$
T(\eta \wedge \xi)=T(\eta) \wedge \xi+\eta \wedge T(\xi)-T(\eta) \wedge T(\xi) .
$$

The operator $(1-T): \mathcal{R} \rightarrow \mathcal{R}_{+}$is an algebra homomorphism, with $\mathcal{R}=\Omega_{X}^{\mathrm{even}}(\log (Y))$ and $\mathcal{R}_{+}=(1-T) \mathcal{R}$. The Birkhoff factorization of a commutative algebra homomorphism $\phi: \mathcal{H} \rightarrow \mathcal{R}$, with $\mathcal{H}$ a commutative Hopf algebra, is given by

$$
\begin{aligned}
& \phi_{+}(\Gamma)=(1-T) \phi(\Gamma) \\
& \phi_{-}(\Gamma)=-T\left(\phi(\Gamma)+\sum \phi_{-}\left(\Gamma^{\prime}\right) \phi\left(\Gamma^{\prime \prime}\right)\right) .
\end{aligned}
$$

Moreover, $\phi_{-}$takes the following form on $\operatorname{ker}(e)=\oplus_{n>0} \mathcal{H}_{n}$ :

$$
\begin{aligned}
\phi_{-}(\Gamma) & =-T(\phi(\Gamma))-\sum_{n=1}^{\infty}(-1)^{n} \sum T\left(\phi\left(\Gamma^{(1)}\right)\right) \phi\left(\Gamma^{(2)}\right) \phi\left(\Gamma^{(3)}\right) \cdots \phi\left(\Gamma^{(n+1)}\right) \\
& =-T(\phi(\Gamma))-\sum_{n=1}^{\infty}(-1)^{n}\left((T \phi) \tilde{*} \phi^{\tilde{*}^{n}}\right)(\Gamma) .
\end{aligned}
$$

Proof. The argument is the same as in the proof of Theorem 6.3 in [21]. It is clear by construction that $T$ is idempotent and the simplified form (4.3) of the Rota-Baxter identity follows by observing that $T(T(\eta) \wedge \xi)=T(\eta) \wedge \xi$ and $T(\eta \wedge T(\xi))=\eta \wedge T(\xi)$ as in Theorem 6.3 in [21]. Then one sees that

$$
(1-T)(\eta) \wedge(1-T)(\xi)=\eta \wedge \xi-T(\eta) \wedge \xi-\eta \wedge T(\xi)+T(\eta) \wedge T(\xi)=\eta \wedge \xi-T(\eta \wedge \xi)
$$

by (4.3). Consider then the Birkhoff factorization. We write $\tilde{\phi}(\Gamma):=\phi(\Gamma)+\sum \phi_{-}\left(\Gamma^{\prime}\right) \phi\left(\Gamma^{\prime \prime}\right)$. The fact that $(1-T)$ is an algebra homomorphism then gives

$$
\begin{gathered}
\phi_{+}(\Gamma)=(1-T)(\tilde{\phi}(\Gamma))=(1-T)\left(\phi(\Gamma)+\sum \phi_{-}\left(\Gamma^{\prime}\right) \phi\left(\Gamma^{\prime \prime}\right)\right) \\
\left.=(1-T)(\phi(\Gamma))+\sum(1-T)\left(\phi_{-}\left(\Gamma^{\prime}\right)\right)(1-T)\left(\phi\left(\Gamma^{\prime \prime}\right)\right)\right),
\end{gathered}
$$

with $(1-T)\left(\phi_{-}\left(\Gamma^{\prime}\right)\right)=-(1-T) T\left(\tilde{\phi}_{-}\left(\Gamma^{\prime}\right)\right)=0$, because $T$ is idempotent. The last statement again follows from Proposition 2.10, since we have $T(T(\eta) \wedge \xi)=T(\eta) \wedge \xi$. 
4.2. Multidimensional residues. In the case of a simple normal crossings divisor $Y \subset X$, we can proceed as discussed in Section 3.2 for the case of a smooth hypersurface. Indeed, as we have seen in Proposition 4.1, we also have in this case a simple pole subtraction $\phi_{+}(\Gamma)=(1-T) \phi(\Gamma)$, even though the negative term $\phi_{-}(\Gamma)$ of the Birkhoff factorization can now be more complicated than in the case of a smooth hypersurface.

The unrenormalized $\phi(\Gamma)$ is a form $\eta=\alpha+\sum_{j} \frac{d f_{j}}{f_{j}} \wedge \beta_{j}$, with $\alpha$ and $\beta_{j}$ holomorphic and $Y_{j}=\left\{f_{j}=0\right\}$ the components of $Y$. Again, if $\alpha=0$ we loose all information about $\phi(\Gamma)$ in our renormalization of the logarithmic form. To avoid this problem, we can again consider, instead of the single renormalized integral $\int_{\sigma} \phi_{+}(\Gamma)$, an additional family of integrals

$$
\int_{\sigma \cap Y_{I}} \operatorname{Res}_{Y_{I}}(\eta)
$$

where $Y_{I}=\cap_{j \in I} Y_{j}$ is an intersection of components of the divisor $Y$ and $\operatorname{Res}_{I}(\eta)$ is the iterated (or multidimensional, or higher) Poincaré residue of $\eta$, in the sense of [25]. These are dual to the iterated Leray coboundaries,

$$
\int_{\sigma \cap Y_{I}} \operatorname{Res}_{Y_{I}}(\eta)=\frac{1}{(2 \pi i)^{n}} \int_{\mathcal{L}_{I}\left(\sigma \cap Y_{I}\right)} \eta
$$

where $\mathcal{L}_{I}=\mathcal{L}_{j_{i}} \circ \cdots \circ \mathcal{L}_{j_{n}}$ for $Y_{I}=Y_{j_{1}} \cap \cdots \cap Y_{j_{n}}$.

If arbitrary intersections $Y_{I}$ of components of $Y$ are all mixed Tate motives, then all these integrals are also periods of mixed Tate motives.

4.3. Saito's logarithmic forms. Given a singular reduced hypersurface $Y \subset X$, a differential form $\omega$ with logarithmic poles along $Y$, in the sense of Saito [58], can always be written in the form $([58,(1.1))$

$$
f \omega=\frac{d h}{h} \wedge \xi+\eta
$$

where $f \in \mathcal{O}_{X}$ defines a hypersurface $V=\{f=0\}$ with $\operatorname{dim}(Y \cap V) \leq \operatorname{dim}(X)-2$, and with $\xi$ and $\eta$ holomorphic forms.

In the following, we use the notation ${ }^{S} \Omega_{X}^{\star}(\log (Y))$ to denote the forms with logarithmic poles along $Y$ in the sense of Saito, to distinguish it from the more restrictive notion of forms with logarithmic poles $\Omega_{X}^{\star}(\log (Y))$ considered above for the normal crossings case.

Following [2], we say that a (reduced) hypersurface $Y \subset X$ has Saito singularities if the modules of logarithmic differential forms and vector fields along $Y$ are free. The condition that $Y \subset X$ has Saito singularities is equivalent to the condition that ${ }^{S} \Omega_{X}^{n}(\log (Y))=\bigwedge^{n}{ }^{S} \Omega_{X}^{1}(\log (Y))$, [58].

Let $Y$ be a hypersurface with Saito singularities and let $\mathcal{M}_{Y}$ denote the sheaf of germs of meromorphic functions on $Y$. Then setting

$$
\operatorname{Res}(\omega)=\left.\frac{1}{f} \xi\right|_{Y}
$$

defines the residue as a morphism of $\mathcal{O}_{X}$-modules, for all $q \geq 1$,

$$
\text { Res : }{ }^{S} \Omega_{X}^{q}(\log (Y)) \rightarrow \mathcal{M}_{Y} \otimes_{\mathcal{O}_{Y}} \Omega_{Y}^{q-1} \text {. }
$$

Unlike the case of smooth hypersurfaces and normal crossings divisors, in the case of more general hypersurfaces with Saito singularities, the Saito residue of forms with logarithmic poles is not a holomorphic form, but only a meromorphic form on $Y$.

For $Y \subset X$ a reduced hypersurface that is quasihomogeneous with Saito singularities, a refinement of (4.7), which we view as the exact sequence

$$
0 \rightarrow \Omega_{X}^{q} \rightarrow{ }^{S} \Omega_{X}^{q}(\log (Y)) \stackrel{\text { Res }}{\rightarrow} \mathcal{M}_{Y} \otimes_{\mathcal{O}_{Y}} \Omega_{Y}^{q-1}
$$


is given in [2, where the image of the Saito Poincaré residue is more precisely identified as $\operatorname{Res}^{S} \Omega_{X}^{q}(\log (Y)) \simeq \omega_{Y}^{q-1}$, where $\omega_{Y}^{\bullet}$ denotes the module of regular meromorphic differential forms in the sense of [8], with $\omega_{Y}^{\bullet} \subset j_{*} j^{*} \Omega_{Y}^{\bullet}$, where $j: S \hookrightarrow Y$ is the inclusion of the singular locus. Namely, it is shown in [2] that one has, for all $q \geq 2$, an exact sequence of $\mathcal{O}_{X}$-modules

$$
0 \rightarrow \Omega_{X}^{q} \rightarrow{ }^{S} \Omega_{X}^{q}(\log (Y)) \stackrel{\text { Res }}{\longrightarrow} \omega_{Y}^{q-1} \rightarrow 0 .
$$

It is natural to ask whether the extraction of polar part from forms with logarithmic poles that we considered here for the case of smooth hypersurfaces and normal crossings divisors extends to more general singular hypersurfaces using Saito's formulation.

Question 4.2. Is there a Rota-Baxter operator $T$ expressed in terms of the Saito residue, in the case of a singular hypersurfaces $Y \subset X$ with Saito singularities?

We describe here a possible approach to this question. We introduce an analog of the Rota-Baxter operator considered above, given by the extraction of the polar part. The "polar part" operator, in this more general case, does not map $\Omega_{X}^{\text {even }}(\log (Y))$ to itself, but we show below that it gives a well defined Rota-Baxter operator of weight -1 on the space of Saito forms ${ }^{S} \Omega_{X}^{\text {even }}(\log (Y))$, and that this operator is a derivation.

Lemma 4.3. The set $S_{Y}:=\{f \mid \operatorname{dim}(\{f=0\} \cap Y) \leq \operatorname{dim}(X)-2\}$ is a multiplicative set. Localization of the Saito forms with logarithmic poles gives $S_{Y}^{-1} S_{\Omega_{X}}(\log (Y))={ }^{S} \Omega_{X}(\log (Y))$.

Proof. We have $V_{12}=\left\{f_{1} f_{2}=0\right\}=\left\{f_{1}=0\right\} \cup\left\{f_{2}=0\right\}$ and

$$
\operatorname{dim}\left(Y \cap V_{12}\right)=\operatorname{dim}\left(\left(Y \cap\left\{f_{1}=0\right\}\right) \cup\left(Y \cap\left\{f_{2}=0\right\}\right)\right) \leq \operatorname{dim}(X)-2,
$$

since $\operatorname{dim}\left(Y \cap\left\{f_{i}=0\right\}\right) \leq \operatorname{dim}(X)-2$ for $i=1,2$. Thus, for any $f_{1}, f_{2} \in S_{Y}$, we have $f_{1} f_{2} \in S_{Y}$. Moreover, we have $1 \in S_{Y}$, hence $S_{Y}$ is a multiplicative set. The localization of ${ }^{S} \Omega_{X}^{\star}(\log (Y))$ at $S_{Y}$ is just ${ }^{S} \Omega_{X}^{\star}(\log (Y))$ itself: in fact, for $\tilde{f}^{-1} \omega \in S_{Y}^{-1} S_{\Omega_{X}^{\star}}(\log (Y))$, with $\tilde{f} \in S_{Y}$ and $\omega \in{ }^{S} \Omega_{X}^{\star}(\log (Y))$, expressed as in (4.5), we have

$$
f \tilde{f}\left(\tilde{f}^{-1} \omega\right)=f \omega=\frac{d h}{h} \wedge \xi+\eta
$$

where $f \tilde{f} \in S_{Y}$, hence $\tilde{f}^{-1} \omega \in S^{S} \Omega_{X}(\log (Y))$.

Given a form $\omega \in{ }^{S} \Omega_{X}^{\star}(\log (Y))$, which we can write as in (4.5), the residue (4.6) is the image under the restriction map $S_{Y}^{-1} \Omega_{X}^{\star} \rightarrow S_{Y}^{-1} \Omega_{Y}^{\star}$ of the form $f^{-1} \xi \in S_{Y}^{-1} \Omega_{X}^{\star}$. Moreover, we have an inclusion $\Omega_{X}^{\star} \hookrightarrow{ }^{S} \Omega_{X}^{\star}(\log (Y))$, which induces a map of the localizations $S_{Y}^{-1} \Omega_{X}^{\star} \hookrightarrow S_{Y}^{-1} S_{\Omega_{X}^{\star}}(\log (Y))=$

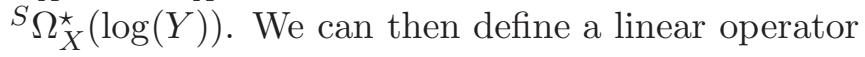

$$
T:{ }^{S} \Omega_{X}^{\star}(\log (Y)) \rightarrow{ }^{S} \Omega_{X}^{\star}(\log (Y)) \wedge S_{Y}^{-1} \Omega_{X}^{\star} \hookrightarrow{ }^{S} \Omega_{X}^{\star}(\log (Y)) \wedge S_{Y}^{-1}{ }^{S} \Omega_{X}^{\star}(\log (Y))={ }^{S} \Omega_{X}^{\star}(\log (Y))
$$

given by

$$
T(\omega)=\frac{d h}{h} \wedge \frac{\xi}{f}, \quad \text { for } \quad f \omega=\frac{d h}{h} \wedge \xi+\eta .
$$

Lemma 4.4. The operator $T$ of (4.9) is a Rota-Baxter operator of weight -1 on ${ }^{S} \Omega_{X}^{\text {even }}(\log (Y))$, which is just given by a derivation, satisfying the Leibnitz rule $T\left(\omega_{1} \wedge \omega_{2}\right)=T\left(\omega_{1}\right) \wedge \omega_{2}+\omega_{1} \wedge T\left(\omega_{2}\right)$.

Proof. Let

Then

$$
f_{1} \omega_{1}=\frac{d h}{h} \wedge \xi_{1}+\eta_{1} \quad f_{2} \omega_{2}=\frac{d h}{h} \wedge \xi_{2}+\eta_{2}
$$

$$
f_{1} f_{2} \omega_{1} \wedge \omega_{2}=\left(\frac{d h}{h} \wedge \xi_{1}+\eta_{1}\right) \wedge\left(\frac{d h}{h} \wedge \xi_{2}+\eta_{2}\right)=\frac{d h}{h} \wedge\left(\xi_{1} \wedge \eta_{2}+(-1)^{p} \eta_{1} \wedge \xi_{2}\right)+\eta_{1} \wedge \eta_{2},
$$


where $\eta_{1} \in \Omega^{p}(X)$. By Lemma 4.3, we know that $f_{1} f_{2} \in S_{Y}$. We have

$$
T\left(\omega_{1} \wedge \omega_{2}\right)=\frac{d h}{h} \wedge\left(\frac{\xi_{1}}{f_{1}} \wedge \frac{\eta_{2}}{f_{2}}+(-1)^{p} \frac{\eta_{1}}{f_{1}} \wedge \frac{\xi_{2}}{f_{2}}\right) .
$$

Since

$$
T\left(\omega_{1}\right)=\frac{d h}{h} \wedge \frac{\xi_{1}}{f_{1}}, \quad \text { and } \quad T\left(\omega_{2}\right)=\frac{d h}{h} \wedge \frac{\xi_{2}}{f_{2}},
$$

we obtain

$$
T\left(\omega_{1}\right) \wedge T\left(\omega_{2}\right)=\frac{d h}{h} \wedge \frac{\xi_{1}}{f_{1}} \wedge \frac{d h}{h} \wedge \frac{\xi_{2}}{f_{2}}=0
$$

Moreover, we have

$$
T\left(\omega_{1}\right) \wedge \omega_{2}=\left(\frac{d h}{h} \wedge \frac{\xi_{1}}{f_{1}}\right) \wedge \frac{d h}{h} \wedge \frac{\xi_{2}}{f_{2}}+\frac{d h}{h} \wedge \frac{\xi_{1}}{f_{1}} \wedge \frac{\eta_{2}}{f_{2}}=\frac{d h}{h} \wedge \frac{\xi_{1}}{f_{1}} \wedge \frac{\eta_{2}}{f_{2}}
$$

with

$$
f_{1} f_{2}\left(T\left(\omega_{1}\right) \wedge \omega_{2}\right)=\frac{d h}{h} \wedge \xi_{1} \wedge \eta_{2}
$$

and similarly,

$$
\omega_{1} \wedge T\left(\omega_{2}\right)=(-1)^{p} \frac{d h}{h} \wedge \frac{\eta_{1}}{f_{1}} \wedge \frac{\xi_{2}}{f_{2}},
$$

hence $T$ satisfies the Leibnitz rule. The operator $T$ also satisfies $T\left(T\left(\omega_{1}\right) \wedge \omega_{2}\right)=T\left(\omega_{1}\right) \wedge \omega_{2}$, and $T\left(\omega_{1} \wedge T\left(\omega_{2}\right)\right)=\omega_{1} \wedge T\left(\omega_{2}\right)$, hence the condition that $T$ is a derivation is equivalent to the condition that it is a Rota-Baxter operator of weight -1 .

Correspondingly, we have

$$
(1-T) \omega=\omega-\frac{d h}{h} \wedge \frac{\xi}{f}=\frac{\eta}{f} \in S_{Y}^{-1} \Omega_{X}^{\text {even }} .
$$

Under the restriction map $S_{Y}^{-1} \Omega_{X}^{\text {even }} \rightarrow S_{Y}^{-1} \Omega_{Y}^{\text {even }}$ we obtain a form $\left.(1-T)(\omega)\right|_{Y}$. It follows that we can define a "subtraction of divergences" operation on $\phi: \mathcal{H} \rightarrow{ }^{S} \Omega_{X}^{\text {even }}(\log (Y))$ by taking $\phi_{+}: \mathcal{H} \rightarrow{ }^{S} \Omega_{X}^{\text {even }}(\log (Y))$ given by $\phi_{+}(a)=\left.(1-T) \phi(a)\right|_{Y}$, for $a \in \mathcal{H}$, which maps $\phi(a)=\omega$ to $\left.(1-T) \omega\right|_{Y}=\left.f^{-1} \eta\right|_{Y}$, where $f \omega=\frac{d h}{h} \wedge \xi+\eta$. While this has subtracted the logarithmic pole along $Y$, it has also created a new pole along $V=\{f=0\}$. Thus, it results again in a meromorphic form. If we consider the restriction to $Y$ of $\phi_{+}(a)=\left.f^{-1} \eta\right|_{Y}$, we obtain a meromorphic form with first order poles along a subvariety $V \cap Y$, which is by hypothesis of codimension at least one in $Y$. Thus, we can conceive of a more complicated renormalization method that progressively subtracts poles on subvarieties of increasing codimension, inside the polar locus of the previous pole subtraction, by iterating this procedure. A more detailed account of this iterative procedure and of possible applications to the setting of renormalization will be discussed elsewhere.

\section{Compactifications of GL $_{n}$ And mOMentum SPACE Feynman integrals}

In this section, we restrict our attention to the case of compactifications of $\mathrm{PGL}_{\ell}$ and of $\mathrm{GL}_{\ell}$ and we use a formulation of the parametric Feynman integrals of perturbative quantum field theory in terms of (possibly divergent) integrals on a cycle in the complement of the determinant hypersurface [4], to obtain a new method of regularization and renormalization. This gives rise to a renormalized integral that is a period of a mixed Tate motive, under certain conditions on the graph and on the intersection of the big cell of the compactification with a divisor $\Sigma_{\ell, g}$. We show that a certain loss of information can occur with respect to the usual physical Feynman integral. 
5.1. The determinant hypersurface. In the following we use the notation $\hat{\mathcal{D}}_{\ell}$ and $\mathcal{D}_{\ell}$, respectively, for the affine and the projective determinant hypersurfaces. Namely, we consider in the affine space $\mathbb{A}^{\ell^{2}}$, identified with the space of all $\ell \times \ell$-matrices, with coordinates $\left(x_{i j}\right)_{i, j=1, \ldots, \ell}$, the hypersurface

$$
\hat{\mathcal{D}}_{\ell}=\left\{\operatorname{det}(X)=0 \mid X=\left(x_{i j}\right)\right\} \subset \mathbb{A}^{\ell^{2}} .
$$

Since $\operatorname{det}(X)=0$ is a homogeneous polynomial in the variables $\left(x_{i j}\right)$, we can also consider the projective hypersurface $\mathcal{D}_{\ell} \subset \mathbb{P}^{\ell^{2}-1}$.

The complement $\mathbb{A}^{\ell^{2}} \backslash \hat{\mathcal{D}}_{\ell}$ is identified with the space of invertible $\ell \times \ell$-matrices, namely with $\mathrm{GL}_{\ell}$.

5.2. The Kausz compactification of $\mathrm{GL}_{n}$. We recall here some basic facts about the Kausz compactification $\mathrm{KGL}_{n}$ of $\mathrm{GL}_{n}$, following [47] and the exposition in $\$ 12$ of [53].

We first recall the Vainsencher compactification [60] of $\mathrm{PGL}_{\ell}$. Let $X_{0}=\mathbb{P}^{\ell^{2}-1}$ be the projectivization of the vector space of square $\ell \times \ell$-matrices. Let $Y_{i}$ be the locus of matrices of rank $i$ and consider the iterated blowups $X_{i}=\mathrm{Bl}_{\bar{Y}_{i}}\left(X_{i-1}\right)$, with $\bar{Y}_{i}$ the closure of $Y_{i}$ in $X_{i-1}$. The $Y_{i}$ are $\mathrm{PGL}_{i^{-}}$ bundles over a product of Grassmannians. It is shown in Theorem 1 and (2.4) of [60] that the $X_{i}$ are smooth, and that $X_{\ell-1}$ is a wonderful compactification of $\mathrm{PGL}_{\ell}$, in the sense of [24]. One denotes by $\overline{\mathrm{PGL}_{\ell}}$ the wonderful compactification of $\mathrm{PGL}_{\ell}$ obtained in this way. We also refer the reader to $\S 12$ of [53] for a quick overview of the main properties of the Vainsencher compactification.

The Kausz compactification [47] of GL $\mathrm{G}_{\ell}$ is similar. One regards $\mathbb{A}^{\ell^{2}}$ as the big cell in $\mathcal{X}_{0}=\mathbb{P}^{\ell^{2}}$. The iterated sequence of blowups is given in this case by setting $\mathcal{X}_{i}=\mathrm{Bl}_{\overline{\mathcal{Y}}_{i-1} \cup \overline{\mathcal{H}}_{i}}\left(\mathcal{X}_{i-1}\right)$, where $\mathcal{Y}_{i} \subset \mathbb{A}^{\ell^{2}}$ are the matrices of rank $i$ and $\mathcal{H}_{i}$ are the matrices at infinity (that is, in $\mathbb{P}^{\ell^{2}-1}=\mathbb{P}^{\ell^{2}} \backslash \mathbb{A}^{\ell^{2}}$ ) of rank $i$. The Kausz compactification is $K \mathrm{GL}_{\ell}=\mathcal{X}_{\ell-1}$. It is shown in Corollary 4.2 of [47] that the $\mathcal{X}_{i}$ are smooth and in Corollary 4.2 and Theorem 9.1 of [47] that the blowup loci are disjoint unions of loci with the following structure: the closure $\overline{\mathcal{Y}}_{i-1}$ in $\mathcal{X}_{i-1}$ is a $K \mathrm{GL}_{i-1}$-bundle over a product of Grassmannians and the closure $\overline{\mathcal{H}}_{i}$ in in $\mathcal{X}_{i-1}$ is a $\overline{\mathrm{PGL}_{i}}$-bundle over a product of Grassmannians. Theorem 9.1 of [47] also shows that these compactifications have a moduli space interpretation. An overview of these properties and of the relation between the Vainsencher and the Kausz compactifications is given in $\S 12$ of [53].

As observed in [53], the Kausz compactification is then the closure of $\mathrm{GL}_{\ell}$ inside the wonderful compactification of $\mathrm{PGL}_{\ell+1}$, see also [43, Chapter 3, §1.4. The compactification $K \mathrm{GL}_{\ell}$ is smooth and projective over $\operatorname{Spec}(\mathbb{Z})$ (Corollary $4.2[47]$ ).

The other property of the Kausz compactification that we will be using in the following is the fact that the complement of the dense open set $\mathrm{GL}_{\ell}$ inside the compactification $K \mathrm{GL}_{\ell}$ is a normal crossing divisor (Corollary 4.2 [47]).

5.3. The virtual motive of the Kausz compactification. We organize the computation of the motive of the Kausz compactification in three subsections, respectively dealing with the virtual motive (Grothendieck class), the numerical motive, and the Chow motive. The main reason for providing separate arguments, instead of giving only the strongest result about the Chow motive, is a pedagogical illustration of the difference between these three levels of motivic structure, where one can see in a very explicit case what is needed to improve from one level to the next, and what are the implications (conditional and unconditional). We begin with the Grothendieck class, which is usually more familiar, especially in the mathematical physics setting.

We use the description recalled above of the Kausz compactification, together with the blowup formula, to check that the virtual motive (class in the Grothendieck ring) of the Kausz compactification is Tate.

Proposition 5.1. Let $K_{0}(\mathcal{V})$ be the Grothendieck ring of varieties (defined over $\mathbb{Q}$ or over $\mathbb{Z}$ ) and let $\mathbb{Z}[\mathbb{L}] \subset K_{0}(\mathcal{V})$ be the Tate subring generated by the Lefschetz motive $\mathbb{L}=\left[\mathbb{A}^{1}\right]$. For all $\ell \geq 1$ the 
class $\left[K \mathrm{GL}_{\ell}\right]$ is in $\mathbb{Z}[\mathbb{L}]$. Moreover, let $\mathcal{Z}_{\ell}$ be the normal crossings divisor $\mathcal{Z}_{\ell}=K \mathrm{GL}_{\ell} \backslash \mathrm{GL}_{\ell}$. Then all the unions and intersections of components of $\mathcal{Z}_{\ell}$ have Grothendieck classes in $\mathbb{Z}[\mathbb{L}]$.

Proof. We use the blowup formula for classes in the Grothendieck ring: if $\tilde{\mathcal{X}}=\operatorname{Bl} \mathcal{Y}(\mathcal{X})$, where $\mathcal{Y}$ is of codimension $m+1$ in $\mathcal{X}$, then the classes satisfy

$$
[\tilde{\mathcal{X}}]=[\mathcal{X}]+\sum_{k=1}^{m}[\mathcal{Y}] \mathbb{L}^{k} .
$$

The Kausz compactification is obtained as an iterated blowup, starting with a projective space, whose class is in $\mathbb{Z}[\mathbb{L}]$ and blowing up at each step a smooth locus that is a bundle over a product of Grassmannians with fiber either a $K \mathrm{GL}_{i}$ or a $\overline{\mathrm{PGL}}_{i}$ for some $i<\ell$. The Grothendieck class of a bundle is the product of the class of the base and the class of the fiber. Classes of Grassmannians (and products of Grassmannians) are in $\mathbb{Z}[\mathbb{L}]$. The classes of the wonderful compactifications $\overline{\mathrm{PGL}}_{i}$ of $\mathrm{PGL}_{i}$ are also in $\mathbb{Z}[\mathbb{L}]$, since it is known that the motive of these wonderful compactifications are mixed Tate (this follows, for instance, from the cell decomposition given in Proposition 4.4. of [41]). Thus, it suffices to assume, inductively, that the classes $\left[K \mathrm{GL}_{i}\right] \in \mathbb{Z}[\mathbb{L}]$ for all $i<\ell$, and conclude via the blowup formula that $\left[K \mathrm{GL}_{\ell}\right] \in \mathbb{Z}[\mathbb{L}]$.

Consider then the boundary divisor $\mathcal{Z}_{\ell}=K \mathrm{GL}_{\ell} \backslash \mathrm{GL}_{\ell}$. The geometry of the normal crossings divisor $\mathcal{Z}_{\ell}$ is described explicitly in $\S 4$ of [47]. It has components $Y_{i}$ and $Z_{i}$, for $0 \leq i \leq \ell$, that correspond to the blowup loci described above. The multiple intersections $\cap_{i \in I} Y_{i} \cap \cap_{j \in J} Z_{j}$ of these components of $\mathcal{Z}_{\ell}$ are described in turn in terms of bundles over products of flag varieties with fibers that are lower dimensional compactifications $K \mathrm{GL}_{i}$ and $\overline{\mathrm{PGL}}_{i}$ and products. Again, flag varieties have cell decompositions, hence their Grothendieck classes are in $\mathbb{Z}[\mathbb{L}]$ and the rest of the argument proceeds as in the previous case. If arbitrary intersections of the components of $\mathcal{Z}_{\ell}$ have classes in $\mathbb{Z}[\mathbb{L}]$ then arbitrary unions and unions of intersections also do by inclusion-exclusion in $K_{0}(\mathcal{V})$.

5.4. The numerical motive of the Kausz compactification. Knowing that the Grothendieck class $\left[K \mathrm{GL}_{\ell}\right]$ is in the Tate subring $\mathbb{Z}[\mathbb{L}] \subset K_{0}(\mathcal{V})$ implies that the motive is of Tate type in the category of pure motives with respect to the numerical equivalence. More precisely, we have the following.

Proposition 5.2. Let $h_{\mathrm{num}}\left(K \mathrm{GL}_{\ell}\right)$ denote the motive of the Kausz compactification $K \mathrm{GL}_{\ell}$ in the category of pure motives over $\mathbb{Q}$, with the numerical equivalence relation. Then $h_{\text {num }}\left(K \mathrm{GL}_{\ell}\right)$ is in the (tensor) subcategory generated by the Tate object. The same is true for arbitrary unions and intersections of the components of the boundary divisor $\mathcal{Z}_{\ell}$ of the compactification.

Proof. The same argument used in Proposition 5.1 can be upgraded at the level of numerical motives. We replace the blowup formula (5.1) for Grothendieck classes with the corresponding formula for motives, which follows (already at the level of Chow motives) from Manin's identity principle, [51]:

$$
h(\tilde{X})=h(X) \oplus \bigoplus_{r=1}^{m} h(Y) \otimes \mathbb{L}^{\otimes r},
$$

with $\tilde{X}=\mathrm{Bl}_{Y}(X)$ the blowup of a smooth subvariety $Y \subset X$ of codimension $m+1$ in a smooth projective variety $X$, and where $\mathbb{L}=h^{2}\left(\mathbb{P}^{1}\right)$ is the Lefschetz motive. Moreover, we use the fact that, for numerical motives, the motive of a locally trivial fibration $X \rightarrow S$ with fiber $Y$ is given by the product

$$
h_{\text {num }}(X)=h_{\text {num }}(Y) \otimes h_{\text {num }}(S),
$$


see Exercise 13.2.2.2 of [5]. The decomposition (5.3) allows us to describe the numerical motives of the blowup loci of the iterated blowup construction of $K \mathrm{GL}_{\ell}$ as products of numerical motives of Grassmannians and of lower dimensional compactifications $K \mathrm{GL}_{i}$ and $\overline{\mathrm{PGL}}_{i}$. The motive of a Grassmannian can be computed explicitly as in [49, already at the level of Chow motives. If $G(d, n)$ denotes the Grassmannian of $d$-planes in $k^{n}$, the Chow motive $h(G(d, n))$ is given by

$$
h(G(d, n))=\bigoplus_{\lambda \in W^{d}} \mathbb{L}^{\otimes|\lambda|}
$$

where

$$
W^{d}=\left\{\lambda=\left(\lambda_{1}, \ldots, \lambda_{d}\right) \in \mathbb{N}^{d} \mid n-d \geq \lambda_{1} \geq \cdots \geq \lambda_{d} \geq 0\right\}
$$

and $|\lambda|=\sum_{i} \lambda_{i}$, see Theorem 2.1 and Lemma 3.1 of [49]. The same decomposition into powers of the Lefschetz motive holds at the numerical level. Moreover, we know (also already for Chow motives) that the motives $h\left(\overline{\mathrm{PGL}}_{i}\right)$ of the wonderful compactifications are Tate (see [41]), and we conclude the argument as in Proposition 5.1 by assuming inductively that the motives $h_{\text {num }}\left(K \mathrm{GL}_{i}\right)$ are Tate, for $i<\ell$. The argument for the loci $\cap_{i \in I} Y_{i} \cap \cap_{j \in J} Z_{j}$ in $\mathcal{Z}_{\ell}$ is analogous.

Remark 5.3. Proposition 5.2 also follows from Proposition 5.1 using the general fact that two numerical motives that have the same class in $K_{0}\left(\operatorname{Num}(k)_{\mathbb{Q}}\right)$ are isomorphic as objects in $\operatorname{Num}(k)_{\mathbb{Q}}$, because of the semi-simplicity of the category of numerical motives [44, together with the existence, for $\operatorname{char}(k)=0$, of a unique ring homomorphism (the motivic Euler characteristic) $\chi_{\text {mot }}: K_{0}\left(\mathcal{V}_{k}\right) \rightarrow$ $K_{0}\left(\operatorname{Num}(k)_{\mathbb{Q}}\right)$, such that $\chi_{\operatorname{mot}}([X])=\left[h_{\text {num }}(X)\right]$, for $X$ a smooth projective variety, see Corollary 13.2.2.1 of [5].

5.5. The Chow motive of the Kausz compactification. Manin's blowup formula (5.2) and the computation of the motive of Grassmannians and of the wonderful compactifications $\overline{\mathrm{PGL}}_{i}$ already hold at the level of Chow motives. However, if we want to extend the argument of Proposition 5.2 to Chow motives, we run into the additional difficulty that one no longer necessarily has the decomposition (5.3) for the motive of a locally trivial fibration. Under some hypotheses on the existence of a cellular structure on the fibers, one can still obtain a decomposition for motives of bundles, and more generally locally trivial fibrations, the fibers of which have cell decompositions with suitable properties, see [46], and also [40], 41], [45], [56]. We obtain an unconditional result on the Chow motive of the Kausz compactification, by analyzing its cellular structure.

Recall that, for $G$ a connected reductive algebraic group and $B$ a Borel subgroup, a spherical variety is a normal algebraic variety on which $G$ acts with a dense orbit of $B$, 14. Spherical varieties can be regarded as a generalization of toric varieties: when $G$ is a torus, one recovers the usual notion of toric variety.

Proposition 5.4. The Chow motive $h\left(K \mathrm{GL}_{\ell}\right)$ of the Kausz compactification is a Tate motive.

Proof. The result follows by showing that $K \mathrm{GL}_{\ell}$ has a cellular structure for all $\ell \geq 1$, which allows us to extend the decomposition of the motive used in Proposition 5.2 from the numerical to the Chow case.

As shown in $\S 3.1$ of [14, it follows from the work of Bialynicki-Birula [1] that any complete, smooth and spherical variety $X$ has a cellular decomposition. This is determined by the decomposition of the spherical variety into $B$-orbits and is obtained by considering a one-parameter subgroup $\lambda: \mathbb{G}_{m} \hookrightarrow X$, such that the set of fixed points $X^{\lambda}$ is finite. The cells are given by

$$
X(\lambda, x)=\left\{z \in X \mid \lim _{t \rightarrow 0} \lambda(t) z=x\right\}, \text { for } x \in X^{\lambda} .
$$

The Kausz compactification $K \mathrm{GL}_{\ell}$ is a smooth toroidal equivariant compactification of $\mathrm{GL}_{\ell}$, see Proposition 1.15 of $\S 3$ of [43] and also Proposition 10.1 and Proposition 12.1 of [53]. In particular, it is a spherical variety (see Proposition 10.1 of [53]), hence it has a cellular structure as above. 
A relative cellular variety, in the sense of [46], is a smooth and proper variety with a decomposition into affine fibrations over proper varieties. The blowup loci of the Kausz compactification $K \mathrm{GL}_{\ell}$ are relative cellular varieties in this sense, since they are bundles over products of Grassmannians, with fiber a lower dimensional compactification $K \mathrm{GL}_{i}$, with $i<\ell$. Using the cell decomposition of the fibers $K \mathrm{GL}_{i}$, we obtain a decomposition of these blowup loci as relative cellular varieties, with pieces of the decomposition being fibrations over a product of Grassmannians, with fibers the cells of the cellular structure of $K \mathrm{GL}_{i}$.

There is an embedding of the category of pure Chow motives in the category of mixed motives, see [5]. By viewing the Chow motives of these blowup loci as elements in the Voevodsky category of mixed motives, Corollary 6.11 of [46] shows that they are direct sums of motives of products of Grassmannians (which are Tate motives), with twists and shifts which depend on the dimensions of the cells of $K \mathrm{GL}_{i}$. We conclude from this that all the blowup loci are Tate motives. We can then repeatedly apply the blowup formula for Chow motives to conclude (unconditionally) that the Chow motive of $K \mathrm{GL}_{\ell}$ is itself a Tate motive. Note that the blowup formula also holds in the Voevodsky category, Proposition 3.5.3 of [61], in the form

$$
\mathfrak{m}\left(\mathrm{Bl}_{Y}(X)\right)=\mathfrak{m}(X) \oplus \bigoplus_{r=1}^{\operatorname{codim}_{X}(Y)-1} \mathfrak{m}(Y)(r)[2 r],
$$

which corresponds to the usual formula of [51] in the case of pure motives, after viewing them as objects in the category of mixed motives. The result can also be obtained, in a similar way, using Theorem 2.10 of [41] instead of Corollary 6.11 of [46].

Remark 5.5. Given the existence of a cellular decomposition of $K \mathrm{GL}_{\ell}$, as above, it is possible to give a quicker proof that the Chow motive is Tate, by using distinguished triangles in the Voevodsky category associated to the inclusions of unions of cells, showing that $\mathfrak{m}\left(K \mathrm{GL}_{\ell}\right)$ is mixed Tate, then using the inclusion of pure motives in the mixed motives to conclude that $h\left(K \mathrm{GL}_{\ell}\right)$ is Tate. In Proposition 5.4 above we chose to maintain the structure of the argument more similar to the cases of the virtual and the numerical motive, for better direct comparison.

Remark 5.6. Notice that a conditional result about the Chow motive would follow directly from Proposition 5.2 or Remark 5.3, if one assumes the Kimura-O'Sullivan finiteness conjecture (or Voevodsky's nilpotence conjecture, which implies it). For the precise statement and implications of the Kimura-O'Sullivan finiteness conjecture, and its relation to Voevodsky's nilpotence, we refer the reader to the survey [6]. By arguing as in Lemma 13.2.1.1 of [5], that would extend the result of Proposition 5.2 to the Chow motive. At the level of Grothendieck classes, the conjecture in fact implies that the $K_{0}$ of Chow motives and the $K_{0}$ of numerical motives coincide, hence one can argue as in Remark 5.3 and conclude that, in order to know that the Chow motive is mixed Tate, it suffices to know that the Grothendieck class is mixed Tate.

5.6. Feynman integrals in momentum space and non-mixed-Tate examples. It was shown in [12] that the parametric form of Feynman integrals in perturbative quantum field theory can be formulated as a (possibly divergent) period integral on the complement of a hypersurface defined by the vanishing of a combinatorial polynomial associated to Feynman graphs. Namely, one writes the (unrenormalized) Feynman amplitudes for a massless scalar quantum field theory as integrals

$$
U(\Gamma)=\frac{\Gamma(n-D \ell / 2)}{(4 \pi)^{\ell D / 2}} \int_{\sigma_{n}} \frac{P_{\Gamma}(t, p)^{-n+D \ell / 2} \omega_{n}}{\Psi_{\Gamma}(t)^{-n+D(\ell+1) / 2}}
$$

where $n=\# E_{\Gamma}$ is the number of internal edges, $\ell=b_{1}(\Gamma)$ is the number of loops, and $D$ is the spacetime dimension. Here we consider the "unregularized" Feynman integral, where $D$ is just the integer valued dimension, without performing the procedure of dimensional regularization that analytically continues $D$ to a complex number. The domain of integration is a simplex $\sigma_{n}=\{t \in$ 
$\left.\mathbb{R}_{+}^{n} \mid \sum_{i} t_{i}=1\right\}$. In the integrand, $\omega_{n}$ is the volume form, and $P_{\Gamma}$ and $\Psi_{\Gamma}$ are polynomials defined as follows. The graph polynomial is defined as

$$
\Psi_{\Gamma}(t)=\sum_{T} \prod_{e \notin T} t_{e}
$$

where the summation is over spanning trees (assuming the graph $\Gamma$ is connected). The polynomial $P_{\Gamma}$ is given by

$$
P_{\Gamma}(t, p)=\sum_{C \subset \Gamma} s_{C} \prod_{e \in C} t_{e}
$$

with the sum over cut-sets $C$ (complements of a spanning tree plus one edge) and with variables $s_{C}$ depending on the external momenta of the graph, $s_{C}=\left(\sum_{v \in V\left(\Gamma_{1}\right)} P_{v}\right)^{2}$, where $\Gamma_{1}$ is one of the connected components after the cut (by momentum conservation, it does not matter which). The variables $P_{v}$ are given by combinations of the external momenta $p=\left(p_{e}\right) \in \mathbb{Q}^{\# E_{\text {ext }}(\Gamma) \cdot D}$, in the form $P_{v}=\sum_{e \in E_{e x t}(\Gamma), t(e)=v} p_{e}$, where $\sum_{e \in E_{e x t}(\Gamma)} p_{e}=0$.

In the range $-n+D \ell / 2 \geq 0$, which includes the log divergent case $n=D \ell / 2$, the Feynman amplitude is therefore the integral of an algebraic differential form defined on the complement of the graph hypersurface $\hat{X}_{\Gamma}=\left\{t \in \mathbb{A}^{n} \mid \Psi_{\Gamma}(t)=0\right\}$. Divergences occur due to the intersections of the domain of integration $\sigma_{n}$ with the hypersurface. Some regularization and renormalization procedure is required to separate the chain of integration from the divergence locus. We refer the reader to [12] (or to [52] for an introductory exposition).

It was originally conjectured by Kontsevich that the graph hypersurfaces $\hat{X}_{\Gamma}$ would always be mixed Tate motives, which would have explained the pervasive occurrence of multiple zeta values in Feynman integral computations observed in [16]. A general result of [10] disproved the conjecture, while more recent results of [18, [19], 29] showed explicit examples of Feynman graphs that give rise to non-mixed-Tate periods.

5.7. Determinant hypersurface and parametric Feynman integrals. In [4 the computation of parametric Feynman integrals was reformulated by replacing the graph hypersurface complement by the complement of the determinant hypersurface.

More precisely, the (affine) graph hypersurface $\hat{X}_{\Gamma}$ is defined by the vanishing of the graph polynomial $\Psi_{\Gamma}$. It follows from the matrix-tree theorem that this polynomial can be written as a determinant

$$
\Psi_{\Gamma}(t)=\operatorname{det} M_{\Gamma}(t)=\sum_{T} \prod_{e \notin T} t_{e}
$$

with $M_{\Gamma}(t)$ the $\ell \times \ell$ matrix

$$
\left(M_{\Gamma}\right)_{k r}(t)=\sum_{i=1}^{n} t_{i} \eta_{i k} \eta_{i r}
$$

where the matrix $\eta$ is given by

$$
\eta_{i k}= \begin{cases} \pm 1 & \text { edge } \pm e_{i} \in \text { loop } \ell_{k} \\ 0 & \text { otherwise }\end{cases}
$$

This definition of the matrix $\eta$ involves the choice of a basis $\left\{\ell_{k}\right\}$ of the first homology $H_{1}(\Gamma ; \mathbb{Z})$ and the choice of an orientation of the edges of the graph, with $\pm e$ denoting the matching/reverse orientation on the edge $e$. The resulting determinant $\Psi_{\Gamma}(t)$ is independent of both choices.

One considers then the map

$$
\Upsilon: \mathbb{A}^{n} \rightarrow \mathbb{A}^{\ell^{2}}, \quad \Upsilon(t)_{k r}=\sum_{i} t_{i} \eta_{i k} \eta_{i r}
$$


that realizes the graph hypersurface as the preimage

$$
\hat{X}_{\Gamma}=\Upsilon^{-1}\left(\hat{\mathcal{D}}_{\ell}\right)
$$

of the determinant hypersurface $\hat{\mathcal{D}}_{\ell}=\left\{\operatorname{det}\left(x_{i j}\right)=0\right\}$.

It is shown in 4 that the map

$$
\Upsilon: \mathbb{A}^{n} \backslash \hat{X}_{\Gamma} \hookrightarrow \mathbb{A}^{\ell^{2}} \backslash \hat{\mathcal{D}}_{\ell}
$$

is an embedding whenever the graph $\Gamma$ is 3-edge-connected with a closed 2-cell embedding of face width $\geq 3$.

Remark 5.7. As discussed in $\S 3$ of [4], the 3-edge-connected condition on graphs can be viewed as a strengthening of the usual 1PI (one-particle-irreducible) condition assumed in physics, since the 1PI condition corresponds to 2-edge-connectivity. In perturbative quantum field theory, one considers 1PI graphs when computing the asymptotic expansion of the effective action. Similarly, one can consider the 2PI effective action (which is related to non-equilibrium phenomena in quantum field theory, see $\S 10.5 .1$ of [57]) and restrict to 3-edge-connected graphs. The condition of having a closed 2-cell embedding of face width $\geq 3$, on the other hand, is a strengthening of the analogous property with face width $\geq 2$, which conjecturally is satisfied for all 2-vertex-connected graphs (strong orientable embedding conjecture, see Conjecture 5.5.16 of [54]). 2-vertex-connectivity is again a natural strengthening of the 1PI condition. A detailed discussion of equivalent formulations and implications of these combinatorial conditions, as well as specific examples of graphs that fail to satisfy them, are given in $\S 3$ of [4].

Let $\mathcal{P}_{\Gamma}(x, p)$ denote a homogeneous polynomial in $x \in \mathbb{A}^{\ell^{2}}$, with $p \in \mathbb{Q}^{\# E_{\text {ext }}(\Gamma) \cdot D}$, with the property that the restriction to the image of the map $\Upsilon=\Upsilon_{\Gamma}$ agrees with the second Symanzik polynomial $P_{\Gamma}$ defined in (5.7),

$$
\left.\mathcal{P}_{\Gamma}(x, p)\right|_{x=\Upsilon(t) \in \Upsilon\left(\mathbb{A}^{n}\right)}=P_{\Gamma}(t, p) .
$$

When the map $\Upsilon_{\Gamma}$ is an embedding, one can, without loss of information, rewrite the parametric Feynman integral as (see Lemma 2.3 of [4])

$$
U(\Gamma)=\int_{\Upsilon\left(\sigma_{n}\right)} \frac{\mathcal{P}_{\Gamma}(x, p)^{-n+D \ell / 2} \omega_{\Gamma}(x)}{\operatorname{det}(x)^{-n+(\ell+1) D / 2}} .
$$

Here $\omega_{\Gamma}(x)$ is an $n$-form on $\mathbb{A}^{\ell^{2}}$ such that the restriction of $\omega_{\Gamma}(x)$ to the subspace $\Upsilon\left(\mathbb{A}^{n}\right)$ satisfies $\omega_{\Gamma}(\Upsilon(t))=\omega_{n}(t)$, with $\omega_{n}$ the volume form on $\mathbb{A}^{n}$. Under the condition that $\Upsilon$ is an embedding, the restriction of the integrand to the image $\Upsilon\left(\sigma_{n}\right)$ then agrees with the original Feynman integral.

The question on the nature of periods is then reformulated in [4 by considering a normal crossings divisor $\hat{\Sigma}_{\Gamma}$ in $\mathbb{A}^{\ell^{2}}$ with $\Upsilon\left(\partial \sigma_{n}\right) \subset \hat{\Sigma}_{\Gamma}$ and considering the motive

$$
\mathfrak{m}\left(\mathbb{A}^{\ell^{2}} \backslash \hat{\mathcal{D}}_{\ell}, \hat{\Sigma}_{\Gamma} \backslash\left(\hat{\Sigma}_{\Gamma} \cap \hat{\mathcal{D}}_{\ell}\right)\right) .
$$

The motive $\mathfrak{m}\left(\mathbb{A}^{\ell^{2}} \backslash \hat{\mathcal{D}}_{\ell}\right)$ of the determinant hypersurface complement belongs to the category of mixed Tate motives (see Theorem 4.1 of [4]), with Grothendieck class

$$
\left[\mathbb{A}^{\ell^{2}} \backslash \hat{\mathcal{D}}_{\ell}\right]=\mathbb{L}^{\left(\begin{array}{l}
\ell \\
2
\end{array}\right)} \prod_{i=1}^{\ell}\left(\mathbb{L}^{i}-1\right) .
$$

However, as shown in [4], the nature of the motive (5.11) is much more difficult to discern, because of the nature of the intersection between the divisor $\hat{\Sigma}_{\Gamma}$ and the determinant hypersurface. Assuming the previous conditions on the graph (3-edge-connectedness with a closed 2-cell embedding of face width at least 3), it is shown in Proposition 5.1 of [4] that one can consider a divisor $\hat{\Sigma}_{\ell, g}$ that 
only depends on $\ell=b_{1}(\Gamma)$ and on the minimal genus $g$ of the surface $S_{g}$ realizing the closed 2-cell embedding of $\Gamma$,

$$
\hat{\Sigma}_{\ell, g}=L_{1} \cup \cdots \cup L_{\left(\begin{array}{c}
f \\
2
\end{array}\right)},
$$

where $f=\ell-2 g+1$ and the irreducible components $L_{1}, \ldots, L_{\left(\begin{array}{l}f \\ 2\end{array}\right)}$ are linear subspaces defined by the equations

$$
\left\{\begin{array}{rll}
x_{i j}=0 & 1 \leq i<j \leq f-1 \\
x_{i 1}+\cdots+x_{i, f-1}=0 & 1 \leq i \leq f-1 .
\end{array}\right.
$$

For a given choice of subspaces $V_{1}, \ldots, V_{\ell}$ of a fixed $\ell$-dimensional space, one defines the variety of frames as

$$
\mathbb{F}\left(V_{1}, \ldots, V_{\ell}\right):=\left\{\left(v_{1}, \ldots, v_{\ell}\right) \in \mathbb{A}^{\ell^{2}} \backslash \hat{\mathcal{D}}_{\ell} \mid v_{k} \in V_{k}\right\} .
$$

In other words, the variety of frames $\mathbb{F}\left(V_{1}, \ldots, V_{\ell}\right)$ consists of the set of $\ell$-tuples $\left(v_{1}, \ldots, v_{\ell}\right)$, with $v_{i} \in V_{i}$, such that $\operatorname{dim} \operatorname{span}\left\{v_{1}, \ldots, v_{\ell}\right\}=\ell$. It is shown in [4 that the motives (5.11) are mixed Tate if the varieties of frames $\mathbb{F}\left(V_{1}, \ldots, V_{\ell}\right)$ are mixed Tate. This question is closely related to the geometry of intersections of unions of Schubert cells in flag varieties and Kazhdan-Lusztig theory.

In this paper we will follow a different approach, which uses the same reformulation of parametric Feynman integrals in momentum space in terms of determinant hypersurfaces, as in [4, but instead of computing the integral in the determinant hypersurface complement, pulls it back to the Kausz compactification of $\mathrm{GL}_{\ell}$, following the model of computations of Feynman integrals in configuration spaces described in [21].

5.8. Cohomology and forms with logarithmic poles. Let $\mathcal{X}$ be a smooth projective variety and $\mathcal{Z} \subset \mathcal{X}$ a divisor. Let $\mathcal{M}_{\mathcal{X}, \mathcal{Z}}^{\star}$ denote, as before, the complex of meromorphic differential forms on $\mathcal{X}$ with poles (of arbitrary order) along $\mathcal{Z}$, and let $\Omega_{\mathcal{X}}^{\star}(\log (\mathcal{Z})$ ) be the complex of forms with logarithmic poles along $\mathcal{Z}$. Let $\mathcal{U}=\mathcal{X} \backslash \mathcal{Z}$ and $j: \mathcal{U} \hookrightarrow \mathcal{X}$ be the inclusion.

Grothendieck's Comparison Theorem, [37], shows that the natural morphism (de Rham morphism)

$$
\mathcal{M}_{\mathcal{X}, \mathcal{Z}}^{\star} \rightarrow R j_{*} \mathbb{C}_{\mathcal{U}}
$$

is a quasi-isomorphism, hence de Rham cohomology $H_{d R}^{\star}(\mathcal{U})$ is computed by the hypercohomology of the meromorphic de Rham complex. In particular, for $\mathcal{U}$ affine, the hypercohomology is not necessary and all classes are represented by closed global differential forms, with hypercohomology replaced by the cohomology of the complex of global sections.

The Logarithmic Comparison Theorem consists of the statement that, for certain classes of divisors $\mathcal{Z}$, the natural morphism

$$
\Omega_{\mathcal{X}}^{\star}(\log (\mathcal{Z})) \rightarrow \mathcal{M}_{\mathcal{X}, \mathcal{Z}}^{\star}
$$

is also a quasi-isomorphism. This is known to hold for simple normal crossings divisors by [25], and for strongly quasihomogeneous free divisors by [20, and for a larger class of locally quasihomogeneous divisors in [42. For our purposes, we will focus only on the case of simple normal crossings divisors.

In combination with Grothendieck's Comparison Theorem, the Logarithmic Comparison Theorem of 25] for a simple normal crossings divisor implies that the de Rham cohomology of the divisor complement is computed by the hypercohomology of the logarithmic de Rham complex,

$$
H_{d R}^{\star}(\mathcal{U}) \simeq \mathbb{H}^{\star}\left(\mathcal{X}, \Omega_{\mathcal{X}}^{\star}(\log \mathcal{Z})\right) .
$$


Remark 5.8. Even under the assumption that the complement $\mathcal{U}$ is affine, the hypercohomology on the right hand side of (5.13) cannot always be replaced by global sections and cohomology. For example, if $\mathcal{X}$ is a smooth projective curve of genus $g$, and $\mathcal{U}$ is the complement of $n$ points in $\mathcal{X}$, then $H_{d R}^{1}(\mathcal{U})$ has dimension $2 g+n-1$, but the dimension of the space of global sections of the sheaf of logarithmic differentials is only $g+n-1$ by Riemann-Roch.

Some direct comparisons between de Rham cohomology $H_{d R}^{\star}(\mathcal{U})$ and the cohomology of the logarithmic de Rham complex are known. We discuss in the coming subsections how these apply to our specific case. Our purpose is to replace the meromorphic form that arises in the Feynman integral computation with a cohomologous form with logarithmic poles along the divisor of the Kausz compactification. In doing so, we need to maintain explicit control of the motive of the variety over which cohomology is taken, and also maintain the algebraic nature of all the differential forms involved.

5.9. Pullback to the Kausz compactification, forms with logarithmic poles, and renormalization. For fixed $D, \ell \in \mathbb{N}$ (respectively the integer spacetime dimension and the loop number) and for assigned external momenta $p \in \mathbb{Q}^{D}$, we now consider the algebraic differential form

$$
\eta_{\Gamma, D, \ell, p}(x):=\frac{\mathcal{P}_{\Gamma}(x, p)^{-n+D \ell / 2} \omega_{\Gamma}(x)}{\operatorname{det}(x)^{-n+(\ell+1) D / 2}} .
$$

For simplicity, we write the above as $\eta_{\Gamma}(x)$. This is defined on the complement of the determinant hypersurface, $\mathbb{A}^{\ell^{2}} \backslash \hat{\mathcal{D}}_{\ell}=\mathrm{GL}_{\ell}$. Thus, by pulling back to the Kausz compactification, we can regard it as an algebraic differential form on

$$
K \mathrm{GL}_{\ell} \backslash \mathcal{Z}_{\ell}=\mathrm{GL}_{\ell}
$$

where $\mathcal{Z}_{\ell}$ is the normal crossings divisor at the boundary of the Kausz compactification.

5.9.1. Cellular decomposition approach. We consider a special case of a simple normal crossings divisor $\mathcal{Z}$ in a smooth projective variety $\mathcal{X}$, under the additional assumption that $\mathcal{X}$ has a cell decomposition. We denote by $\left\{X_{\alpha, i}\right\}$ the finite collection of cells of dimension $i$, and in particular we simply write $X_{\alpha}=X_{\alpha, \operatorname{dim} \mathcal{X}}$ for the top dimensional cells.

Proposition 5.9. Let $\mathcal{Z} \subset \mathcal{X}$ be a pair as above, with $\left\{X_{\alpha}\right\}$ the top dimensional cells of the cellular decomposition. Given a meromorphic form $\eta \in \mathcal{M}_{\mathcal{X}, \mathcal{Z}}^{m}$, there exist forms $\beta^{(\alpha)}$ on $X_{\alpha}$ with logarithmic poles along the normal crossings divisor $\mathcal{Z}$, such that

$$
\left[\beta^{(\alpha)}\right]=\left[\left.\eta\right|_{X_{\alpha}}\right] \in H_{d R}^{*}\left(X_{\alpha} \backslash \mathcal{Z}\right) .
$$

Proof. Lemma 2.5 of [20] shows that the Logarithmic Comparison Theorem is equivalent to the statement that, for all Stein open sets $\mathcal{V} \subset \mathcal{X}$, there are isomorphisms $H^{\star}\left(\Gamma\left(\mathcal{V}, \Omega_{\mathcal{X}}^{\star}(\log \mathcal{Z})\right)\right) \simeq$ $H_{d R}^{\star}(\mathcal{V} \backslash \mathcal{Z})$. Namely, the hypercohomology in the Logarithmic Comparison Theorem can be replaced by cohomology of the complex of sections, when restricted to Stein open sets.

Remark 5.10. The forms $\beta^{(\alpha)}$ do not match consistently on the closures of the cells $X_{\alpha}$, because of nontrivial Cech cocycles, hence they are not restrictions of a unique form with logarithmic poles $\beta$ defined on all of $\mathcal{X}$. In particular, the forms $\beta^{(\alpha)}$ obtained in this way depend on the cellular decomposition used.

Lemma 5.11. Let $\mathcal{Z} \subset \mathcal{X}$ and $\left\{X_{\alpha}\right\}$ be as above, and suppose given a meromorphic form $\eta \in$ $\mathcal{M}_{\mathcal{X}, \mathcal{Z}}^{N}$, with $N=\operatorname{dim} \mathcal{X}$, and an $N$-chain $\sigma \subset \mathcal{X}$ with $\partial \sigma \subset \Sigma$, for a divisor $\Sigma$ in $\mathcal{X}$. After performing a pole subtraction on the logaritmic forms on each cell $X_{\alpha}$ one can replace the integral $\int_{\Sigma} \eta$ with a renormalized version

$$
\int_{\sigma} \beta^{+}:=\sum_{\alpha} \int_{X_{\alpha} \cap \sigma} \beta^{(\alpha),+},
$$


where $\beta^{(\alpha)++}$ is a simple pole subtraction on $\beta^{(\alpha)}$. The integral (5.16) is a sum of periods of motives $\mathfrak{m}\left(X_{\alpha}, X_{\alpha} \cap \Sigma\right)$. The information contained in the subtracted polar part is recovered by the Poincaré residues

$$
\int_{\sigma \cap \mathcal{Z}_{I}} \operatorname{Res}_{\mathcal{Z}_{I}}(\beta):=\sum_{\alpha} \int_{\sigma \cap \mathcal{Z}_{I} \cap X_{\alpha}} \operatorname{Res}_{\mathcal{Z}_{I}}\left(\beta^{(\alpha)}\right)
$$

along the intersections of components $\mathcal{Z}_{I}=Z_{i_{1}} \cap \cdots \cap Z_{i_{k}}, I=\left\{i_{1}, \ldots, i_{k}\right\}$ of the divisor $\mathcal{Z}$. These are sums of periods of the motives $\mathfrak{m}\left(\mathcal{Z}_{I} \cap X_{\alpha}\right)$.

Proof. Given the cell decomposition as above, we can write the integral as

$$
\int_{\sigma} \eta=\left.\sum_{\alpha} \int_{X_{\alpha} \cap \sigma} \eta\right|_{X_{\alpha}}=\sum_{\alpha} \int_{X_{\alpha} \cap \sigma} \beta^{(\alpha)},
$$

where each $\left.\eta\right|_{X_{\alpha}}$ is replaced by the cohomologous $\beta^{(\alpha)}$ with logarithmic poles. After performing a pole subtraction on each $\beta^{(\alpha)}$ we obtain holomorphic forms $\beta^{(\alpha),+}$, hence the resulting integral is a period of $\mathfrak{m}\left(X_{\alpha}, X_{\alpha} \cap \Sigma\right)$. For the relation between polar subtraction and the Poincaré residues, see the discussion in 3.2 and 4.2 above.

In both (5.16) and (5.17), we use the notation on the left-hand-side, with a global integral and a global form $\beta$, purely as a formal shorthand notation for the sum of the integrals on the cells of the $\beta^{(\alpha)}$, since the latter are not restrictions of a global form $\beta$.

Remark 5.12. Notice that the resulting integral (5.16) obtained in this way can be identified with a period of $\mathfrak{m}(\mathcal{X}, \Sigma)$ only in the case where the forms $\beta^{(\alpha),+}$ are restrictions $\beta^{(\alpha),+}=\left.\beta^{+}\right|_{X_{\alpha}}$ of a single holomorphic form $\beta^{+}$on $\mathcal{X}$. More generally, the resulting (5.16) is only a sum of periods of the motives $\mathfrak{m}\left(X_{\alpha}, X_{\alpha} \cap \Sigma\right)$.

Remark 5.13. If the cellular decomposition of $\mathcal{X}$ has a single top dimensional cell $X$, then a unique form with logarithmic poles $\beta \in \Omega_{X}^{\star}(\log \mathcal{Z})$, satisfying $\left[\left.\eta\right|_{X}\right]=[\beta] \in H_{d R}^{\star}(X \backslash \mathcal{Z})$, suffices to regularize the integral $\int_{\sigma} \eta$, with regularized value $\int_{\sigma \cap X} \beta^{+}$.

As we discussed in Proposition 5.4, the Kausz compactification is a spherical variety (Proposition 1.15 of $\S 3$ of [43] and also Propositions 9.1, 10.1 and 12.1 of [53]), hence it has a cellular decomposition ( $\S 3.1$ of [14]) into cells $X(\lambda, x)$ as in (5.5). Thus, we can apply the procedure described above, to regularize the integral $\int_{\Upsilon(\sigma)} \eta_{\Gamma}$. While this regularization procedure depends on the choice of the cell decomposition, the construction of [14] for spherical varieties provides a cellular structure that is intrinsically defined by the orbit structure of $K \mathrm{GL}_{\ell}$ and is quite naturally reflecting its geometry. We can then perform a renormalization procedure based on the pole subtraction procedure for forms with logarithmic poles described above.

Corollary 5.14. The cell decomposition $\{X(\lambda, x)\}$ of $K \mathrm{GL}_{\ell}$ has a single big cell $X$. Given $\eta_{\Gamma}=$ $\eta_{\Gamma, D, \ell, p}$ as in (5.14), there is a form $\beta_{\Gamma}=\beta_{\Gamma, D, \ell, p}$ on the big cell $X$, with logarithmic poles along $\mathcal{Z}_{\ell}$, such that $\left[\left.\eta_{\Gamma}\right|_{X}\right]=\left[\beta_{\Gamma}\right] \in H_{d R}^{\star}(X \backslash \mathcal{Z})$. Applying the Birkhoff factorization for forms with logarithmic poles to $\beta_{\Gamma}$, we obtain a renormalized integral of the form

$$
R(\Gamma)=\int_{\tilde{\Upsilon}\left(\sigma_{n}\right) \cap X} \beta_{\Gamma, D, \ell, p}^{+},
$$

where $\beta_{\Gamma}^{+}$is a simple pole subtraction on $\beta_{\Gamma}$.

Proof. As mentioned in Proposition 5.4, the spherical variety $K \mathrm{GL}_{\ell}$ is a smooth toroidal equivariant compactification of $\mathrm{GL}_{\ell}$ (Proposition 1.15 of $\S 3$ of [43] and Propositions 9.1 and 12.1 of [53]). By 
$\S 2.3$ of [15] and Proposition 9.1 of [53], it then follows that there is just one big cell $X$. We can then write the integral as

$$
\int_{\tilde{\Upsilon}\left(\sigma_{n}\right)} \eta_{\Gamma}=\left.\int_{X \cap \tilde{\Upsilon}\left(\sigma_{n}\right)} \eta_{\Gamma}\right|_{X}
$$

where $\tilde{\Upsilon}\left(\sigma_{n}\right)$ is the pullback to $K \mathrm{GL}_{\ell}$ of the domain of integration $\Upsilon\left(\sigma_{n}\right)$.

Let $\mathcal{H}$ be the Hopf algebra of Feynman graphs. The morphism $\phi: \mathcal{H} \rightarrow \mathcal{M}_{X, \mathcal{Z}_{\ell} \cap X}^{*}$ assigns to a Feynman graph $\Gamma$ a meromorphic differential form $\beta_{\Gamma}=\beta_{\Gamma, D, \ell, p}$ with logarithmic poles along $\mathcal{Z}_{\ell}$ satisfying $\left[\left.\eta_{\Gamma}\right|_{X}\right]=\left[\beta_{\Gamma}\right] \in H_{d R}^{\star}(X \backslash \mathcal{Z})$.

We then perform the Birkhoff factorization, and we denote by $\beta_{\Gamma}^{+}$the regular differential form on $X \subset K \mathrm{GL}_{\ell}$ given by $\phi^{+}(\Gamma)=\beta_{\Gamma}^{+}$. Since we only have logarithmic poles, by Proposition 4.1 the operation becomes a simple pole subtraction and we have $\beta_{\Gamma}^{+}=(1-T) \beta_{\Gamma}$.

If we assume, as above, that the external momenta $p$ in the polynomial $\mathcal{P}_{\Gamma}(x, p)$ are rational, then the form $\eta_{\Gamma}=\eta_{\Gamma, D, \ell, p}(x)$ is an algebraic differential form defined over $\mathbb{Q}$, hence we can also assume that the form with logarithmic poles $\beta_{\Gamma}$ is also defined over $\mathbb{Q}$.

In addition to the integral (5.19), one also has the collection of the iterated Poincaré residues along the intersections of components of the divisor $\mathcal{Z}_{\ell}$. Namely, for any $\mathcal{Z}_{I, \ell}=\cap_{j \in I} Z_{j, \ell}$, with $Z_{j, \ell}$ the components of $\mathcal{Z}_{\ell}$, we have the additional integrals

$$
\mathcal{R}(\Gamma)_{I}=\int_{\tilde{\Upsilon}\left(\sigma_{n}\right) \cap \mathcal{Z}_{I, \ell} \cap X} \operatorname{Res}_{\mathcal{Z}_{I}}\left(\beta_{\Gamma}\right) .
$$

5.9.2. Griffiths-Schmid approach. A global replacement of $\eta_{\Gamma}$ by a single form $\beta_{\Gamma, D, \ell, p}$ on $K \mathrm{GL}_{\ell}$ with logarithmic poles along $\mathcal{Z}_{\ell}$ can be obtained if we use the $\mathcal{C}^{\infty}$-logarithmic de Rham complex instead of the algebraic or analytic one.

Proposition 5.15. Consider the class $\left[\eta_{\Gamma}\right]$ in the analytic de Rham cohomology $H_{d R}^{*}\left(\mathrm{GL}_{\ell} ; \mathbb{C}\right)$. There is a $\mathcal{C}^{\infty}$-form $\beta_{\Gamma}^{\infty}$ on $K \mathrm{GL}_{\ell}$ with logarithmic poles along $\mathcal{Z}_{\ell}$ such that

$$
\left[\beta_{\Gamma}^{\infty}\right]=\left[\eta_{\Gamma}\right] \in H_{d R}^{*}\left(K \mathrm{GL}_{\ell} \backslash \mathcal{Z}_{\ell} ; \mathbb{C}\right)=H_{d R}^{*}\left(\mathrm{GL}_{\ell} ; \mathbb{C}\right) .
$$

Applying the Birkhoff factorization yields a renormalized integral

$$
R^{\infty}(\Gamma)=\int_{\tilde{\Upsilon}\left(\sigma_{n}\right)} \beta_{\Gamma, D, \ell, p}^{\infty,+},
$$

where $\beta_{\Gamma}^{\infty,+}$ is a simple pole subtraction on $\beta_{\Gamma}^{\infty}$, and iterated residues

$$
\mathcal{R}^{\infty}(\Gamma)_{I}=\int_{\tilde{\Upsilon}\left(\sigma_{n}\right) \cap \mathcal{Z}_{I, \ell}} \operatorname{Res}_{\mathcal{Z}_{I}}\left(\beta_{\Gamma}^{\infty}\right) .
$$

Proof. For $\mathcal{X}$ a complex smooth projective variety and $\mathcal{Z}$ a simple normal crossings divisor, let $\Omega_{\mathcal{C}^{\infty}(\mathcal{X})}(\log \mathcal{Z})$ be the $\mathcal{C}^{\infty}$-logarithmic de Rham complex. The Griffiths-Schmid theorem (Proposition 5.14 of [36]) shows that there is an isomorphism $H_{d R}^{*}(\mathcal{U})=H^{*}\left(\Omega_{\mathcal{C}^{\infty}(\mathcal{X})}(\log \mathcal{Z})\right)$.

Remark 5.16. With the Griffiths-Schmid theorem one looses the algebraicity of differential forms. Namely, the forms $\beta_{\Gamma}^{\infty}$ and $\beta_{\Gamma}^{\infty,+}$ are only smooth and not algebraic or analytic differential forms. Even if the resulting form $\beta_{\Gamma}^{\infty,+}$, after pole subtraction, can then be replaced by an algebraic de Rham form in the same cohomology class in $H_{d R}^{*}\left(K \mathrm{GL}_{\ell} ; \mathbb{C}\right)$, it will remain, in general, only a form with $\mathbb{C}$-coefficients and not one defined over $\mathbb{Q}$. Thus, following this approach one obtains a consistent renormalization procedure, but one can lose control on the description of the resulting integrals as periods of motives defined over a number field. 
5.9.3. The Hodge filtration approach. There is another case in which a form can be replaced globally by a cohomologous one with logarithmic poles on the complement of a normal crossings divisor, while only using algebraic or analytic forms. Indeed, there is a particular piece of the de Rham cohomology that is always realized by global sections of the (algebraic) logarithmic de Rham complex. This is the piece $F^{n} H_{d R}^{n}(\mathcal{U})$ of the Hodge filtration of Deligne's mixed Hodge structure, with $n=\operatorname{dim} \mathcal{X}$. This Hodge filtration on $\mathcal{U}$ is given by

$$
F^{p} H_{d R}^{k}(\mathcal{U})=\operatorname{Im}\left(\mathbb{H}^{k}\left(\mathcal{X}, \Omega_{\mathcal{X}}^{\geq p}(\log \mathcal{Z})\right) \rightarrow \mathbb{H}^{k}\left(\mathcal{X}, \Omega_{\mathcal{X}}^{\star}(\log \mathcal{Z})\right)\right)
$$

Proposition 5.17. Let $\mathcal{X}$ be a smooth projective variety with $N=\operatorname{dim} \mathcal{X}$, and let $\mathcal{Z}$ be a simple normal crossings divisor with affine complement $\mathcal{U}=\mathcal{X} \backslash \mathcal{Z}$. Then, for $n \leq N$, the Hodge filtration satisfies

$$
H^{0}\left(\mathcal{X}, \Omega_{\mathcal{X}}^{n}(\log \mathcal{Z})\right)=F^{n} H_{d R}^{n}(\mathcal{U}) / F^{n+1} H_{d R}^{n}(\mathcal{U}) .
$$

In the case where $n=N$ the right-hand-side is reduced to $F^{N} H_{d R}^{N}(\mathcal{U})$.

Proof. The Hodge filtration $F^{p} H_{d R}^{k}(\mathcal{U})$ is induced by the naive filtration on $\Omega_{\mathcal{X}}^{\star}(\log \mathcal{Z})$. Recall that (see Theorem 8.21 and Proposition 8.25 of [62]) the spectral sequence of a filtration $F$ on a complex $K^{\star}$ that comes from a double complex $K^{p, q}$, with

$$
F^{p} K^{n}=\oplus_{r \geq p, r+s=n} K^{r, s}
$$

has terms

$$
\begin{gathered}
E_{0}^{p, q}=\operatorname{Gr}_{p}^{F} K^{p+q}=F^{p} K^{p+q} / F^{p+1} K^{p+q}=K^{p, q} \\
E_{1}^{p, q}=H^{p+q}\left(\operatorname{Gr}_{p}^{F} K^{\star}\right)=H^{q}\left(K^{p, \star}\right) \\
E_{\infty}^{p, q}=\operatorname{Gr}_{p}^{F} H^{p+q}\left(K^{\star}\right) .
\end{gathered}
$$

The spectral sequence above, applied to the Hodge filtration $F^{p} H_{d R}^{k}(\mathcal{U})$, is referred to as the Frölicher spectral sequence. It has

$$
\begin{gathered}
E_{1}^{p, q}=H^{q}\left(\mathcal{X}, \Omega_{\mathcal{X}}^{p}(\log \mathcal{Z})\right) \\
E_{\infty}^{p, q}=F^{p} H_{d R}^{p+q}(\mathcal{U}) / F^{p+1} H_{d R}^{p+q}(\mathcal{U}) .
\end{gathered}
$$

In particular, $E_{1}^{n, 0}=H^{0}\left(\mathcal{X}, \Omega_{\mathcal{X}}^{n}(\log \mathcal{Z})\right)$ and $E_{\infty}^{n, 0}=F^{n} H_{d R}^{n}(\mathcal{U}) / F^{n+1} H_{d R}^{n}(\mathcal{U})$. When $n=N=$ $\operatorname{dim} \mathcal{X}$, the term $F^{N+1} H_{d R}^{N}(\mathcal{U})$ vanishes for dimensional reasons.

Deligne proved in [25] (see also the formulation of the result given in Theorem 8.35 of [62]) that, in the case where $\mathcal{Z}$ is a normal crossings divisor, the Frölicher spectral sequence of the Hodge filtration degenerates at the $E_{1}$ term. Thus, in particular, we obtain (5.25).

Corollary 5.18. Given a meromorphic form $\eta$ with $[\eta] \in F^{n} H_{d R}^{n}\left(\mathrm{GL}_{\ell}\right) / F^{n+1} H_{d R}^{n}\left(\mathrm{GL}_{\ell}\right)$, with $n \leq \ell^{2}=\operatorname{dim} K \mathrm{GL}_{\ell}$, there is a form $\beta$ on $K \mathrm{GL}_{\ell}$ with logarithmic poles along the normal crossings divisor $\mathcal{Z}_{\ell}$, such that

$$
[\beta]=[\eta] \in H_{d R}^{n}\left(K \mathrm{GL}_{\ell} \backslash \mathcal{Z}_{\ell}\right)=H_{d R}^{n}\left(\mathrm{GL}_{\ell}\right) .
$$

Then after pole subtraction one obtains

$$
\int_{\tilde{\Upsilon}\left(\sigma_{n}\right)} \beta^{+}
$$

which is a period of $\mathfrak{m}\left(K \mathrm{GL}_{\ell}, \Sigma_{\ell, g}\right)$. 
In this case also, in addition to the integral (5.27), we also have the iterated residues (which in this case exist globally),

$$
\int_{\tilde{\Upsilon}\left(\sigma_{n}\right) \cap \mathcal{Z}_{I, \ell}} \operatorname{Res}_{\mathcal{Z}_{I}}(\beta) .
$$

In general, it is difficult to estimate where the form $\eta_{\Gamma}$ lies in the Hodge filtration. One can give an estimate, based on the relation between the filtration by order of pole and the Hodge filtration, but it need not be accurate because exact forms can cancel higher order poles. The same issue was discussed, in the original formulation in the graph hypersurface complement, in $§ 9.2$ and Proposition 9.8 of [13].

Let $\mathcal{X}$ be a smooth projective variety and $\mathcal{Z} \subset \mathcal{X}$ a simple normal crossings divisor. As before, let $\mathcal{M}_{\mathcal{Z}, \mathcal{X}}^{\star}$ denote the complex of meromorphic differential forms on $\mathcal{X}$ with poles (of arbitrary order) along $\mathcal{Z}$. This complex has a filtration $P^{\star} \mathcal{M}_{\mathcal{Z}, \mathcal{X}}^{\star}$ by order of poles (polar filtration), where $P^{k} \mathcal{M}_{\mathcal{Z}, \mathcal{X}}^{m}$ consists of the $m$-forms with pole of order at most $m-k+1$, if $m-k \geq 0$ and zero otherwise. Deligne showed in $\S I$ I.3, Proposition 3.13 of [26] and Proposition 3.1.11 of [25], that the filtration induced on the subcomplex $\Omega_{\mathcal{X}}^{\star}(\log \mathcal{Z})$ by the polar filtration on $\mathcal{M}_{\mathcal{Z}, \mathcal{X}}^{\star}$ is the naive filtration (that is, the Hodge filtration), and that the natural morphism

$$
\left(\Omega_{\mathcal{X}}^{\star}(\log \mathcal{Z}), F^{\star}\right) \rightarrow\left(\mathcal{M}_{\mathcal{Z}, \mathcal{X}}^{\star}, P^{\star}\right)
$$

is a filtered quasi-isomorphism. In particular (Theorem 2 of [27]) the image of $\mathbb{H}^{\star}\left(\mathcal{X}, P^{k} \mathcal{M}_{\mathcal{X}, \mathcal{Z}}^{\star}\right)$ inside $H_{d R}^{\star}(\mathcal{U})$ contains $F^{k} H_{d R}^{\star}(\mathcal{U})$. This means that we can use the order of pole to obtain at least an estimate of the position of $\left[\eta_{\Gamma}\right]$ in the Hodge filtration. We need to compute the order of pole of the pullback of the form $\eta_{\Gamma}$ along the blowups in the construction of the compactification $K \mathrm{GL}_{\ell}$.

Proposition 5.19. For a graph $\Gamma$ with $n=\# E_{\Gamma}$ and $\ell=b_{1}(\Gamma)$, such that $n \geq \ell-2$, and with spacetime dimension $D \in \mathbb{N}$, the position of $\left[\eta_{\Gamma}\right]$ in the Hodge filtration $F^{k} H_{d R}^{n}\left(\mathrm{GL}_{\ell}\right)$ is estimated by $k \geq n-(\ell-1)(-n+(\ell+1) D / 2)+(\ell-1)^{2}$.

Proof. At the first step in the construction of the compactification $K \mathrm{GL}_{\ell}$ we blow up the locus of matrices of rank one. We need to compare the order of vanishing of $\operatorname{det}(x)^{-n+(\ell+1) D / 2}$ along this locus, with the order of zero acquired by the form $\omega_{\Gamma}$ along the exceptional divisor of this blowup. The determinant vanishes at order $\ell-1$ on that stratum. The form $\omega_{\Gamma}$, on the other hand, acquires a zero of order $c-1$ where $c$ is the codimension of the blowup locus. This can be seen in a local model: when blowing up a locus $L=\left\{z_{1}=\cdots=z_{c}=0\right\}$ in $\mathbb{C}^{N}$, the local coordinates $w_{i}$ in the blowup can be taken as $w_{i} w_{c}=z_{i}$ for $i<c$ and $w_{i}=z_{i}$ for $i \geq c$, with $E=\left\{w_{c}=0\right\}$ the exceptional divisor. Then for $n \geq c$, and a form $d z_{1} \wedge \cdots \wedge d z_{n}$, the pullback satisfies

$$
\pi^{*}\left(d z_{1} \wedge \cdots \wedge d z_{n}\right)=d\left(w_{c} w_{1}\right) \wedge \cdots \wedge d\left(w_{c} w_{c-1}\right) \wedge d\left(w_{c}\right) \wedge \cdots \wedge d\left(w_{n}\right)=w_{c}^{c-1} d w_{1} \wedge \cdots \wedge d w_{n} .
$$

The codimension of the locus of rank one matrices is $c=(\ell-1)^{2}$. Thus, when performing the first blowup in the construction of $K \mathrm{GL}_{\ell}$, the pullback of the form $\eta_{\Gamma}$ acquires a pole of order $(\ell-1)(-n+(\ell+1) D / 2)-(\ell-1)^{2}+1$ along the exceptional divisor. Further blowups do not alter this pole order, hence we can estimate that the pullback of the $n$-form $\eta_{\Gamma}$ to the Kausz compactification is in the term $P^{k}$ of the polar filtration, with $n-k+1=(\ell-1)(-n+(\ell+1) D / 2)-(\ell-1)^{2}+1$. Taking into account the possibility of reductions of the order of pole, due to cancellations coming from exact forms, we obtain an estimate for the position in the polar and in the Hodge filtration, with $k \geq n-(\ell-1)(-n+(\ell+1) D / 2)+(\ell-1)^{2}$. 
5.10. Nature of the period. We then discuss the nature of the period obtained by the evaluation of (5.27). We need a preliminary result. We define a mixed Tate configuration $Y$ in an ambient variety $X$ as follows.

Definition 5.20. Let $X$ be a smooth projective variety and $Y \subset X$ a divisor with irreducible components $\left\{Y_{i}\right\}_{i=1}^{N}$. Let $\mathcal{C}_{Y}=\left\{Y_{I}=Y_{i_{1}} \cap \cdots \cap Y_{i_{k}} \mid I=\left(i_{1}, \ldots, i_{k}\right), k \leq N\right\}$. Then $Y$ is a mixed Tate configuration if all unions $Y_{I_{1}} \cup \cdots \cup Y_{I_{r}}$ of elements of the set $\mathcal{C}_{Y}$ have motives $\mathfrak{m}\left(Y_{I_{1}} \cup \cdots \cup Y_{I_{r}}\right)$ contained in the Voevodsky derived category of mixed Tate motives.

Remark 5.21. Note that in Definition 5.20 we do not require that $Y$ is necessarily a normal crossings divisor. However, in the case of the boundary divisor $\mathcal{Z}_{\ell}$ of $K \mathrm{GL}_{\ell}$, we will use in Proposition 5.29 the fact that it is also normal crossings, in addition to satisfying the condition of Definition 5.20 (see Lemma 5.23).

Let $\Sigma_{\ell, g}$ be the proper transform of the divisor given by the projective version of $\hat{\Sigma}_{\ell, g}$ described in (5.12), defined by the same equations.

Lemma 5.22. The divisor $\Sigma_{\ell, g}$ is a mixed Tate configuration.

Proof. By (5.12), $\Sigma_{\ell, g}$ and any arbitrary union of components are hyperplane arrangements. It is known from [9] that motives of hyperplane arrangements are mixed Tate, see also $\$ 1.7 .1-1.7 .2$ and $\S 3.1 .1$ of [30], where the computation of the motive in the Voevodsky category can be obtained in terms of Orlik-Solomon models. Using a characterization of the mixed Tate condition in terms of eigenvalues of Frobenius, the mixed Tate nature of hyperplane arrangements was also proved in Proposition 3.1.1 of [48. The mixed Tate property can be seen very explicitly at the level of the virtual motive. In fact, the Grothendieck class of an arrangement $A$ in $\mathbb{P}^{n}$ is explicitly given (Theorem 1.1. of [3]) by

$$
[A]=\left[\mathbb{P}^{n}\right]-\frac{\chi_{\hat{A}}(\mathbb{L})}{\mathbb{L}-1}
$$

where $\chi_{\hat{A}}(t)$ is the characteristic polynomial of the associated central arrangement $\hat{A}$ in $\mathbb{A}^{n+1}$. It then follows by inclusion-exclusion in the Grothendieck ring that all unions and intersections of components of $A$ are mixed Tate.

Lemma 5.23. The boundary divisor $\mathcal{Z}_{\ell}$ of the Kausz compactification $K \mathrm{GL}_{\ell}$ is a mixed Tate configuration.

Proof. The motives of unions of intersections of components of $\mathcal{Z}_{\ell}$ can be described in terms of motives of bundles over products of flag varieties with fibers that are lower dimensional compactifications $K \mathrm{GL}_{i}$ and $\overline{\mathrm{PGL}}_{i}$, and we proved in Proposition 5.4 (see also Propositions 5.1 and 5.2) that all these motives are Tate, hence the condition of Definition 5.20 is satisfied.

Proposition 5.24. When the form $\beta_{\Gamma, D, \ell, p}$ on the big cell extends to a logarithmic form in $\Omega_{K \mathrm{GL}_{\ell}}^{\star}\left(\log \mathcal{Z}_{\ell}\right)$, the integral $R(\Gamma)=\int_{\tilde{\Upsilon}\left(\sigma_{n}\right)} \beta_{\Gamma, D, \ell, p}^{+}$is a period of a mixed Tate motive.

Proof. In the globally defined case, this is an integral of an algebraic differential form defined on the compactification $K \mathrm{GL}_{\ell}$, hence a genuine period, in the sense of algebraic geometry, of $K \mathrm{GL}_{\ell}$. By Proposition 5.4, we know that the Chow motive $h\left(K \mathrm{GL}_{\ell}\right)$ is Tate. We also know from Lemma 5.22 that the motive $\mathfrak{m}\left(\Sigma_{\ell, g}\right)$ is mixed Tate. Under the embedding of pure motives into mixed motives we obtain objects $\mathfrak{m}\left(K \mathrm{GL}_{\ell}\right)$ and $\mathfrak{m}\left(\Sigma_{\ell, g}\right)$ in the derived category of mixed Tate motives, $\mathcal{D} \mathcal{M} \mathcal{M}(\mathbb{Q})$, that is, the smallest triangulated subcategory of the Voevodsky triangulated category of mixed motives $\mathcal{D} \mathcal{M}(\mathbb{Q})$ containing all the Tate objects $\mathbb{Q}(n)$. It then follows that the relative motive $\mathfrak{m}\left(K \mathrm{GL}_{\ell}, \Sigma_{\ell, g}\right)$ is also mixed Tate, as it sits in a distinguished triangle in the Voevodsky triangulated category, where the other two terms are mixed Tate. 
Remark 5.25. In the proof of Proposition 5.24 here above, we viewed the motive $\mathfrak{m}\left(K \mathrm{GL}_{\ell}, \Sigma_{\ell, g}\right)$ as an element in the derived category $\mathcal{D} \mathcal{M} \mathcal{T} \mathcal{M}(\mathbb{Q})$ of mixed Tate motives. All the varieties we are considering are defined over a number field, in fact over $\mathbb{Q}$. In the number field case, an abelian category of mixed Tate motives can be constructed as the heart of a $t$-structure in $\mathcal{D} \mathcal{M} \mathcal{T} \mathcal{M}(\mathbb{Q})$ : this is possible because the Beilinson-Soulé vanishing conjecture holds over a number field, see [50]. We denote by $\mathcal{M T} \mathcal{M}(\mathbb{Q})$ this abelian category of mixed Tate motives. To obtain objects in $\mathcal{M T} \mathcal{M}(\mathbb{Q})$ one applies the cohomology functor with respect to the $t$-structure. For example, for a projective space $\mathbb{P}^{n}$, one has the motive $\mathfrak{m}\left(\mathbb{P}^{n}\right)=\mathbb{Q}(0) \oplus \mathbb{Q}(-1)[2] \oplus \cdots \oplus \mathbb{Q}(-n)[2 n]$ in $\mathcal{D} \mathcal{M T} \mathcal{M}(\mathbb{Q})$; its cohomology with respect to the $t$-structure is $h_{2 i}\left(\mathfrak{m}\left(\mathbb{P}^{n}\right)\right)=\mathbb{Q}(-i)$, which is an object in $\mathcal{M T} \mathcal{M}(\mathbb{Q})$, while the shifts are not. In the following, with an abuse of notation, we will write the motive simply as $\mathfrak{m}\left(K \mathrm{GL}_{\ell}, \Sigma_{\ell, g}\right)$, and more generally $\mathfrak{m}\left(K \mathrm{GL}_{\ell} \backslash A, B\right)$ for the cases considered in Proposition 5.29, although when we refer to the motive in $\mathcal{M T} \mathcal{M}(\mathbb{Q})$ what we are really considering are the pieces of the cohomology with respect to the $t$-structure, and in particular, for the conclusion about the period, the piece that corresponds to the degree of the differential form.

Proposition 5.26. Let $\beta_{\Gamma, D, \ell, p}$ be the form with logarithmic poles on the top cell $X$ of the cellular decomposition of $K \mathrm{GL}_{\ell}$, as in Corollary 5.14. If the motive $\mathfrak{m}\left(\Sigma_{\ell, g} \cap X\right)$ is mixed Tate, then the integral $R(\Gamma)=\int_{\tilde{\Upsilon}\left(\sigma_{n}\right)} \beta_{\Gamma, D, \ell, p}^{+}$is a period of a mixed Tate motive.

Proof. Using distinguished triangles in the Voevodsky category, we see that, if the motive $\mathfrak{m}\left(\Sigma_{\ell, g} \cap\right.$ $X)$ is mixed Tate, then the motive $\mathfrak{m}\left(X, \Sigma_{\ell, g} \cap X\right)$ also is, since the big cell has $\mathfrak{m}(X)=\mathbb{L}^{\ell^{2}}$. The result then follows, since the integral is by construction a period of the motive $\mathfrak{m}\left(X, \Sigma_{\ell, g} \cap X\right)$.

Remark 5.27. The central difficulty in the approach of [4, which was to analyze the nature of the motive of $\mathfrak{m}\left(\Sigma_{\ell, g} \cap \mathcal{D}_{\ell}\right)$, is here replaced by the problem of identifying the nature of the motive $\mathfrak{m}\left(\Sigma_{\ell, g} \cap X\right)$, where $X$ is the big cell of $K \mathrm{GL}_{\ell}$.

It may seem at first that we have simply substituted the problem of understanding for which range of $(\ell, g)$ the intersection of the divisor $\Sigma_{\ell, g}$ with $\mathrm{GL}_{\ell}$ remains mixed Tate, with the very similar problem of when the intersection of $\Sigma_{\ell, g}$ with the big cell $X$ of $K \mathrm{GL}_{\ell}$ remains mixed Tate. However, this reformulation makes it possible to use the explicit description of the cells $X(\lambda, x)$ of spherical varieties in terms of limits as in (5.5), to analyze this question. We do not discuss this further in the present paper, and we simply state it as a question.

Question 5.28. Let $X$ be the big cell of the cellular decomposition (5.5) of $K_{\mathrm{GL}_{\ell}}$ and let $\Sigma_{\ell, g}$ be the divisor described above. For which pairs $(\ell, g)$ is the motive $\mathfrak{m}\left(\Sigma_{\ell, g} \cap X\right)$ mixed Tate?

One defines the category $\mathcal{M T} \mathcal{M}(\mathbb{Z})$ of mixed Tate motives over $\mathbb{Z}$ as mixed Tate motives in $\mathcal{M T} \mathcal{M}(\mathbb{Q})$ that are unramified over $\mathbb{Z}$. An object of $\mathcal{M T} \mathcal{M}(\mathbb{Q})$ is unramified over $\mathbb{Z}$ if and only if, for any prime $p$, there exists a prime $\ell \neq p$ such that the $\ell$-adic realization is unramified at $p$, see Proposition 1.8 of [28]. In the following statement our notation for the motives is meant as in Remark 5.25 above.

Proposition 5.29. The motives $\mathfrak{m}\left(K \mathrm{GL}_{\ell}\right)$ are unramified over $\mathbb{Z}$. More generally, if $A$ and $B$ are unions of two disjoint sets of components of the boundary divisor $\mathcal{Z}_{\ell}$ of the compactification $K \mathrm{GL}_{\ell}$, the motives $\mathfrak{m}\left(K \mathrm{GL}_{\ell} \backslash A, B\right)$ are unramified over $\mathbb{Z}$.

Proof. This question can be approached in a way analogous to our previous discussion of the Chow motive, namely using the description of $K \mathrm{GL}_{\ell}$ as an iterated blowup and the properties of the divisor of the compactification. The argument is similar to the one used in Theorem 4.1 and Proposition 4.3 of [35] to prove the analogous statement for the moduli spaces $\overline{\mathcal{M}}_{0, n}$ of rational curved with marked points. There, it is shown that $\overline{\mathcal{M}}_{0, n}$ is unramified over $\mathbb{Z}$ by showing that the 
combinatorics of the normal crossings divisor of the compactification is not altered by reductions $\bmod p$, see Definition 4.2 of [35]. For a pair $(\mathcal{X}, \mathcal{Z})$, with $\mathcal{Z} \subset \mathcal{X}$ a normal crossing divisor, the condition that the reduction mod $p$ does not alter the combinatorics means that $\mathcal{X}$ and all the strata of $\mathcal{Z}$ are smooth over $\mathbb{Z}_{p}$ and the reduction $\bmod p$ gives a bijection from the strata of $\mathcal{Z}$ to those of the special fiber (see Definition 4.2 of 35] and Definition 3.9 of [34]). In our case we have $\mathcal{X}=K \mathrm{GL}_{\ell}$, with $\mathcal{Z}=\mathcal{Z}_{\ell}$ the boundary divisor of the Kausz compactification. As we showed in $\S \$ 5.3$, 5.4, and 5.5, the motive $\mathfrak{m}\left(K \mathrm{GL}_{\ell}\right)$ is a Tate motive. More generally, the motives $\mathfrak{m}\left(K \mathrm{GL}_{\ell} \backslash A, B\right)$ are mixed Tate over $\mathbb{Q}$ : this can be seen as in Proposition 3.6 of [34, using Lemma 5.23, which shows that $\mathcal{Z}_{\ell}$ is both a normal crossings divisor and a mixed Tate configuration in the sense of Definition 5.20. This implies, by the argument of Proposition 3.6 of [34], that all the motives $\mathfrak{m}\left(K \mathrm{GL}_{\ell} \backslash A, B\right)$ are mixed Tate over $\mathbb{Q}$. To check the condition that the reduction map preserves the combinatorics of $\left(K \mathrm{GL}_{\ell}, \mathcal{Z}_{\ell}\right)$, first note that both $K \mathrm{GL}_{\ell}$ and the strata of the normal crossing divisor $\mathcal{Z}_{\ell}$ are smooth over $\mathbb{Z}$, by Corollary 4.2 [47]. Moreover, the description of the Kausz compactification and of the strata of its boundary divisor given in Theorems 9.1 and 9.3 of [47] also holds over fields of characteristic $p$ and is compatible with reduction, so that the set of strata is matched under the reduction map. The argument of Proposition 4.3 of [35] showing that the $\ell$-adic realization is then unramified, for all $\ell$ with $\ell \neq p$, is based on the argument of Proposition 3.10 of [34]. Following this reasoning, the cohomologies $H^{*}(\mathcal{X} \backslash A, B)$ can be computed using a simplicial resolution $\mathcal{S}_{\bullet}(\mathcal{X} \backslash A, B)$, whose simplexes correspond to unions of intersections of components of the divisor. The argument of Proposition 3.10 of 34 then shows that the reduction map applied to the simplicial schemes $\mathcal{S}_{\bullet}(\mathcal{X} \backslash A, B)$ induces an isomorphism in étale cohomology, $H_{e t}^{*}\left(\overline{\mathcal{X}} \backslash \bar{A}, \bar{B}, \mathbb{Q}_{\ell}\right) \simeq H_{e t}^{*}\left(\overline{\mathcal{X}}^{0} \backslash \bar{A}^{0}, \bar{B}^{0}, \mathbb{Q}_{\ell}\right)$, where $\overline{\mathcal{X}}=\mathcal{X} \otimes_{\mathbb{Z}_{p}} \mathbb{Q}_{p}$ and $\mathcal{X}^{0}$ is the special fiber of the reduction. This shows that the étale realization is unramified for $\ell \neq p$. By Proposition 1.8 of [28] this means that the motives $\mathfrak{m}\left(K \mathrm{GL}_{\ell} \backslash A, B\right)$ are mixed Tate over $\mathbb{Z}$.

Remark 5.30. Given that the unramified condition holds, one can conclude from Brown's theorem [17] and the previous Proposition 5.24 (and Proposition 5.26, when $\mathfrak{m}\left(\Sigma_{\ell, g} \cap X\right)$ is mixed Tate) that the integral (5.27) is a $\mathbb{Q}\left[\frac{1}{2 \pi i}\right]$-linear combination of multiple zeta values.

5.11. Comparison with Feynman integrals. The result obtained in this way clearly differs from the usual computation of Feynman integrals, where the methods used are based on regularization and pole subtraction of the integral (dimensional regularization, cutoff, zeta regularization, etc.) There are several reasons behind this difference, which we now discuss briefly.

In the usual physical renormalization non-mixed-Tate periods are known to occur, [18, [19]. In the setting we discussed here, the only possible source of non-mixed-Tate cases is the motive of the intersection $\Sigma_{\ell, g} \cap X$, where $X$ is the big cell of the Kausz compactification $K \mathrm{GL}_{\ell}$. In particular, this locus is the same for all graphs with fixed loop number $\ell$ and fixed genus $g$. However, in the usual physical renormalization, not all graphs with the same $\ell$ and $g$ have periods of the same nature, as one can see from the examples analyzed in [29], [59].

There is loss of information in mapping the computation of the Feynman integral from the complement of the graph hypersurface (as in [12, [18, [19]) to the complement of the determinant hypersurface (as in [4), when the combinatorial conditions on the graph recalled in 5.7 are not satisfied. Explicit examples of graphs that violate those conditions are given in $\S 3$ of [4]. In such cases the map (5.9) need not be an embedding, hence part of the information contained in the Feynman integral calculation (5.6) will be lost in passing to (5.10).

However, this type of loss of information does not affect some of the cases where non-mixed Tate motives are known to appear in the momentum space Feynman amplitude.

Example 5.31. Let $\Gamma$ be the graph with 14 edges that gives a counterexample to the Kontsevich polynomial countability conjecture, in Section 1 of [29]. The map $\Upsilon: \mathbb{A}^{n} \rightarrow \mathbb{A}^{\ell^{2}}$ of (5.9) has 
$n=\# E(\Gamma)=14$ and $\ell=b_{1}(\Gamma)=7$. Let $\Upsilon_{i}$ denote the composition of the map $\Upsilon$ with the projection onto the $i$-th row of the matrix $M_{\Gamma}$ of (5.8). In order to check if the embedding condition for $\Upsilon$ is satisfied, we know from Lemma 3.1 of [4 that it suffices to check that $\Upsilon_{i}$ is injective for $i$ ranging over a set of loops such that every edge of $\Gamma$ is part of a loop in that set. This can then be checked by computer verification for the matrix $M_{\Gamma}$ of this particular graph.

The example above is a log divergent graph in dimension four. It is known to give a non-mixed Tate contribution with the usual method of computation of the Feynman integral, 229, [18]. The same verification method we used for this case can be applied to the other currently known explicit counterexamples in [29], [18], [59], [19].

Even for integrals (including the example above) where the map (5.9) is an embedding, the regularization and renormalization procedure described here, using the Kausz compactification and subtraction of residues for forms with logarithmic poles, is not equivalent to the usual renormalization procedures of the regularized integrals. For instance, our regularized form (hence our regularized integral) can be trivial in cases where the usual regularization and renormalization would give a non-trivial result. This may occur if the form $\beta$ with logarithmic poles happens to have a nontrivial residue, but a trivial holomorphic part $\beta^{+}$.

In such cases, part of the information loss coming from pole subtraction on the differential form is compensated by keeping track of the residues. However, in our setting these also deliver only mixed Tate periods, so that even when this information is included, one still loses the richer structure of the periods arising from other methods of regularization and renormalization, adopted in the physics literature.

Acknowledgment. The authors are very grateful to the anonymous referee for many very detailed and helpful comments and suggestions that greatly improved the paper. The first author was partially supported by NSF grants DMS-1007207, DMS-1201512, and PHY-1205440.

\section{REFERENCES}

[1] A.G. Aleksandrov, Multidimensional residue theory and the logarithmic de Rham complex, J. of Singularities, Vol. 5 (2012) 1-18.

[2] A.G. Aleksandrov, Nonisolated hypersurface singularities, in "Theory of Singularities and its Applications" (V.I. Arnold, Ed.) Advances in Soviet Mathematics, Vol. 1, American Mathematical Society, 1990, pp. 211-246.

[3] P. Aluffi, Grothendieck classes and Chern classes of hyperplane arrangements, Int. Math. Res. Not. IMRN (2013) no. 8, $1873 ? 1900$.

[4] P. Aluffi, M. Marcolli, Parametric Feynman integrals and determinant hypersurfaces, Adv. Theor. Math. Phys. 14 (2010), no. 3, 911-963.

[5] Y. André, Une introduction aux motifs, Société Mathématique de France, 2004.

[6] Y. André, Motifs de dimension finie, Séminaire Bourbaki, 2003-04, Exp. N. 929 viii, Astérisque N.299 (2005) $115-145$.

[7] F.V. Atkinson, Some aspects of Baxter's functional equation, J. Math. Anal. Appl. 7 (1963) 1-30.

[8] D. Barlet, Le faisceau $\omega_{X}^{\bullet}$ sur un espace analytique $X$ de dimension pure, in "Fonctions de plusieurs variables complexes, III (Sém. François Norguet, 1975-1977)", pp. 187-204, Lecture Notes in Math. Vol.670, Springer,1978.

[9] A.A. Beilinson, A.B. Goncharov, V.V. Schechtman, A.N. Varchenko, Aomoto dilogarithms, mixed Hodge structures and motivic cohomology of pairs of triangles on the plane, in "The Grothendieck Festschrift", Vol. I, pp.135-172, Progr. Math., Vol.86, Birkhäuser, 1990.

[10] P. Belkale, P. Brosnan, Matroids, motives, and a conjecture of Kontsevich, Duke Math. J. 116 (2003) N. 1, $147-188$.

[11] A. Bialynicki-Birula, Some theorems on actions of algebraic groups, Annals of Math. 98 (1973) $480-497$.

[12] S. Bloch, H. Esnault, D. Kreimer, On motives associated to graph polynomials, Comm. Math. Phys. 267 (2006), no. $1,181-225$.

[13] S. Bloch, D. Kreimer, Mixed Hodge structures and renormalization in physics, Commun. Number Theory Phys. Vol.2 (2008) N.4, 637-718. 
[14] M. Brion, Spherical varieties, Proceedings of the International Congress of Mathematicians, Vol. 1, 2 (Zürich, 1994), pp.753-760, Birkhäuser, 1995.

[15] M. Brion, D. Luna, Sur la structure locale des variétés sphériques, Bull. Soc. Math. France 115 (1987) $211-226$.

[16] D. Broadhurst, D. Kreimer, Association of multiple zeta values with positive knots via Feynman diagrams up to 9 loops, Phys. Lett. B 393 (1997) N.3-4, 403-412.

[17] F. Brown, Mixed Tate motives over $\mathbb{Z}$, Ann. of Math. (2) 175 (2012), no. 2, 949-976.

[18] F. Brown, D. Doryn, Framings for graph hypersurfaces, arXiv:1301.3056.

[19] F. Brown, O. Schnetz, A K3 in $\phi^{4}$, Duke Math. J. 161 (2012), no. 10, 1817-1862.

[20] F.J. Castro-Jiménez, L. Narváez-Macarro, D. Mond, Cohomology of the complement of a free divisor, Trans. Amer. Math. Soc., Vol.348 (1996) N.8, 3037-3049.

[21] O. Ceyhan, M. Marcolli, Algebraic renormalization and Feynman integrals in configuration spaces, Adv. Theor. Math. Phys. 18 (2014) N.2, 469-511.

[22] A. Connes, D. Kreimer, Renormalization in quantum field theory and the Riemann-Hilbert problem. I. The Hopf algebra structure of graphs and the main theorem, Comm. Math. Phys. 210 (2000), no. 1, 249-273.

[23] A. Connes, M. Marcolli, Noncommutative Geometry, quantum fields and motives, Colloquium Publications, Vol.55, American Mathematical Society, 2008.

[24] C. De Concini, C. Procesi, Complete symmetric varieties, in "Invariant theory" (Montecatini, 1982), pp. 1-44, Lecture Notes in Math. 996, Springer, 1983.

[25] P. Deligne, Théorie de Hodge. II, Inst. Hautes Études Sci. Publ. Math. No. 40 (1971), 5-57.

[26] P. Deligne, Equations différentielles à points singuliers réguliers, Lecture Notes in Mathematics, Vol. 163. Springer-Verlag, 1970.

[27] P. Deligne, A. Dimca, Filtrations de Hodge et par l'ordre du pôle pour les hypersurfaces singulières, Ann. Sci. École Norm. Sup. (4) 23 (1990), no. 4, 645-656.

[28] P. Deligne, A.B. Goncharov, Groupes fondamentaux motiviques de Tate mixte, Ann. Scient. Éc. Norm. Sup., Vol.38 (2005) 1-56.

[29] D. Doryn, On one example and one counterexample in counting rational points on graph hypersurfaces, Lett. Math. Phys. 97 (2011), no. 3, 303-315.

[30] C. Dupont, Periods of hyperplane arrangements and motivic coproduct, Ph.D. Thesis, Université Pierre et Marie Curie - Paris VI, 2014. https://tel.archives-ouvertes.fr/tel-01083524/document

[31] K. Ebrahimi-Fard, L. Guo, D. Kreimer, Integrable renormalization. II. The general case, Ann. Henri Poincaré 6 (2005), no. 2, 369-395.

[32] K. Ebrahimi-Fard, L. Guo, D. Kreimer, Spitzer's Identity and the Algebraic Birkhoff Decomposition in pQFT, J. Phys. A: Math. Gen., 37, (2004) 11037-11052.

[33] A.B. Goncharov, Multiple polylogarithms and mixed Tate motives, arXiv:math/0103059

[34] A.B. Goncharov, Periods and mixed Tate motives, arXiv:math/0202154v2.

[35] A.B. Goncharov, Yu.I. Manin, Multiple $\zeta$-motives and moduli spaces $\overline{\mathcal{M}}_{0, n}$, Compositio Math., Vol.140 (2004) $1-14$.

[36] P. Griffiths, W. Schmid, Recent developments in Hodge theory: a discussion of techniques and results, in "Discrete subgroups of Lie groups and applicatons to moduli" (Internat. Colloq., Bombay, 1973), pp. 31-127. Oxford Univ. Press, Bombay, 1975.

[37] A. Grothendieck, On the de Rham cohomology of algebraic varieties, Pub. Math. I.H.E.S. 29 (1966) 95-105.

[38] L. Guo, An Introduction to Rota-Baxter Algebra, International Press and High Education Press, 2012.

[39] L. Guo, Algebraic Birkhoff decomposition and its applications. In: Automorphic Forms and the Langlands Program. International Press, 2008, pp. 283-323.

[40] S. Habibi, The motive of a bundle, PhD Thesis, Università degli Studi di Milano, 2012.

[41] S. Habibi, M.E.A. Rad, On the motive of G-bundles, arXiv:1112.4110v3.

[42] M.P. Holland, D. Mond Logarithmic differential forms and the cohomology of the complement of a divisor, Math. Scand. 83 (1998), No.2, 235-254.

[43] M. Huruguen, Compactification d'espaces homogènes sphériques sur un corps quelconque, PhD Thesis, Université de Grenoble, 2011. https://tel.archives-ouvertes.fr/tel-00716402/document

[44] U. Jannsen, Motives, numerical equivalence, and semi-simplicity, Invent. Math. 107 (1992) no. 3, $447-452$.

[45] B. Kahn, Motivic Cohomology of smooth geometrically cellular varieties, in "Algebraic K-theory, Seattle, 1997", pp. 149-174, Proceedings of Symposia in Pure Mathematics, vol. 67, American Mathematical Society, 1999.

[46] N. Karpenko, Cohomology of relative cellular spaces and isotropic flag varieties, St. Petersburg Math. J., Vol.12 (2001) N. 1, 1-50.

[47] I. Kausz, A modular compactification of the general linear group, Documenta Math. 5 (2000) 553-594.

[48] M. Kisin, G. Lehrer, Eigenvalues of Frobenius and Hodge Numbers, Pure and Applied Math. Quarterly, Vol.2 (2006) N.2, 199-219.

[49] B. Köck, Chow motif and higher Chow theory of G/P, Manuscripta Math. 70 (1991), no. 4, 363-372. 
[50] M. Levine, Tate motives and the vanishing conjectures for algebraic $K$-theory, in "Algebraic $K$-theory and algebraic topology" (Lake Louise, AB, 1991), 167-188, NATO Adv. Sci. Inst. Ser. C Math. Phys. Sci., 407, Kluwer Acad. Publ., 1993.

[51] Yu.I. Manin, Correspondences, motifs and monoidal transformations, Mat. Sb. (N.S.) 77 (119) $1968475-507$.

[52] M. Marcolli, Feynman motives, World Scientific, 2010.

[53] J. Martens, M. Thaddeus, Compactifications of reductive groups as moduli stacks of bundles, Compositio Math. 152 (2016) 62-98.

[54] B. Mohar, C. Thomassen, Graphs on surfaces, Johns Hopkins University Press, 2001.

[55] D. Mond, Notes on logarithmic vector fields, logarithmic differential forms and free divisors, preprint, 2012.

[56] C. Pompeyo-Gutiérrez, Chow motive of a locally trivial fibration, arXiv:1205.4287.

[57] J. Rammer, Quantum field theory of non-equilibrium states, Cambridge University Press, 2007.

[58] K. Saito, Theory of logarithmic differential forms and logarithmic vector fields, J. Fac. Sci. Univ. Tokyo Sect. IA Math. 27 (1980), no. 2, 265-291.

[59] O. Schnetz, Quantum field theory over $\mathbb{F}_{q}$, Electron. J. Comb.18 (2011) N.1, P102.

[60] I. Vainsencher, Complete collineations and blowing up determinantal ideals, Math. Ann. 267 (1984), no. 3, 417432.

[61] V. Voevodsky, Triangulated categories of motives over a field, in "Cycles, transfer and motivic homology theories", Annals of Mathematical Studies, Vol. 143, Princeton Univ. Press, 2000, pp. 188-238.

[62] C. Voisin, Hodge theory and complex algebraic geometry. I, Cambridge Studies in Advanced Mathematics, Vol.76, Cambridge University Press, 2007.

Department of Mathematics, Division of Mathematics, Physics and Astronomy, California Institute of Technology, 1200 E. California Blvd. Pasadena, CA 91125, USA

E-mail address: matilde@caltech.edu

E-mail address: xni@caltech.edu 